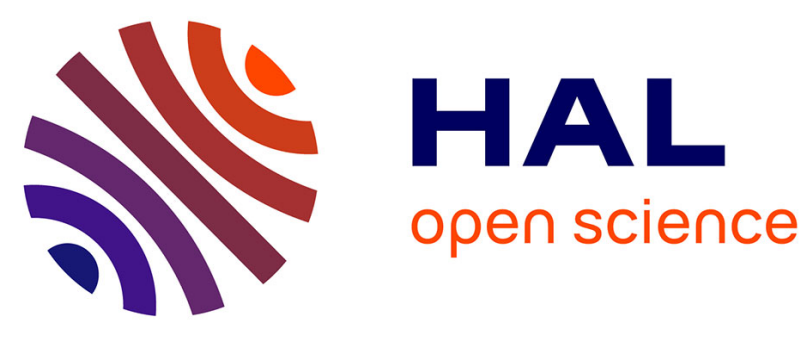

\title{
Inventory of sedimentary organic matter in modern wetland (Marais Vernier, Normandy, France) as source-indicative tools to study Holocene alluvial deposits (Lower Seine Valley, France).
}

David Sebag, Christian Di Giovanni, S. Ogier, V. Mesnage, Fatima

Laggoun-Défarge, A. Durand

\section{To cite this version:}

David Sebag, Christian Di Giovanni, S. Ogier, V. Mesnage, Fatima Laggoun-Défarge, et al.. Inventory of sedimentary organic matter in modern wetland (Marais Vernier, Normandy, France) as source-indicative tools to study Holocene alluvial deposits (Lower Seine Valley, France).. International Journal of Coal Geology, 2006, 67, pp.1-16. 10.1016/j.coal.2005.08.002 . hal-00022634

\section{HAL Id: hal-00022634 \\ https://hal-insu.archives-ouvertes.fr/hal-00022634}

Submitted on 24 May 2006

HAL is a multi-disciplinary open access archive for the deposit and dissemination of scientific research documents, whether they are published or not. The documents may come from teaching and research institutions in France or abroad, or from public or private research centers.
L'archive ouverte pluridisciplinaire HAL, est destinée au dépôt et à la diffusion de documents scientifiques de niveau recherche, publiés ou non, émanant des établissements d'enseignement et de recherche français ou étrangers, des laboratoires publics ou privés.

\section{(1) (1) $\$$}

Distributed under a Creative Commons Attribution - NonCommercial - NoDerivatives 44.0 


\title{
Inventory of sedimentary organic matter in modem wetland (Marais Vemier, Nomandy, France) as source-indicative tools to study Holocene alluvial deposits (Lower Seine Valley, France)
}

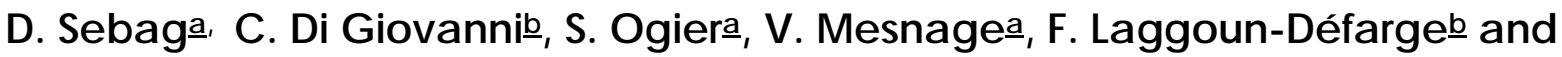 \\ A. Duranda
}

aUMR 6143 CNRS, Morphodyna mique Continentale et Côtière, Dépa rtement de Géologie, Université de Rouen, 76821 Mont Saint Aignan Cedex, France bISTO, UMR 6113 CNRS, Bâtiment Géosc iences, Université d'O rléa ns, BP 6759, 45067 Orléans Cedex 2, France

Keywords: Wetland deposits; Organic matter; Palynofacies; Optical markers; Palaeoenvironment; Holocene

\section{Abstract}

Palynofacies' analysis was developed for petroleum exploration, but it is now commonly used to differentiate the major constituents of the sedimentary organic matter (SOM) in Postglacial deposits. Our work aimed at studying the SOM distribution in a present wetland of the Seine estuary (Vernier Marsh) in order to provide comparative elements to interpret the composition of SOM in the Lower Seine Valley alluvial infilling. In the Vernier Marsh, the distribution of major constituents is heterogeneous. Thus, for each depositional environment studied (soils, drainage ditches, channels, ponds), the composition of SOM has been characterized by using two ternary diagrams (“Amorphous OM/Preserved Phytoclasts/Transformed Phytoclasts” and "Opaque/Amorphous/Gelified Particles”; OP/AP/GP). This approach allowed us to define four optical indices that can be used to estimate the relative abundances of principal organic fractions in mixed deposits based on the origin of SOM (aquatic/terrestrial), its source areas (vegetation, soils, river), and controls of early diagenetic changes (aerobic/anaerobic conditions, advanced oxidation). "Amorphous OM contents" increase from terrestrial environments to fluvial deposits and evaluate the abundance of planktonic contribution. "Preserved / transformed phytoclasts ratio" decreases from sites of production to those of deposition indicating the degree of degradation of terrestrial materials. "OP contents" allow one to define fluvial (high values) and palustrine (low values) environments. "GP / AP ratio" allows one to discern soil (low values) and plant (high values) supplied areas. Finally, these optical compositional indices are measured in Holocene deposits and used to complete palaeoenvironmental reconstructions. 


\section{Introduction}

Wetlands are among the most important ecosystems that include specific biotopes (Whigham, 1999). In addition, they are favourable for organic matter (OM) preservation and for peat accumulation (Clymo, 1983). The latter is the subject of numerous works aiming to understand the possible influence of human activities on natural environments (Barber et al., 2003) and the function of the global carbon cycle (Gorham, 1991 and Malmer, 1992). The study of sedimentary $\mathrm{OM}$ is also the subject of a considerable amount of work done through geochemical analyses. In order to trace the origin, transport and fate of organic matter from diverse sources, isotopic and/or molecular marker (biomarker) analyses have been applied. Although each method has proven quite successful, there are limitations. For example, the characterization of natural and anthropogenic OM by bulk chemical methods provides an averaged signal which can be difficult to interpret because of its heterogeneous nature. However, a large part of this heterogeneity appears at the micrometer or nanometer scale (Tyson, 1995). So, the micromorphological characterization of OM constitutes a useful and complementary method for determining the nature of OM and its origin, and for understanding its diagenetic evolution (Laggoun-Défarge et al., 1995 and Cohen et al., 1999). One mean of analysing OM, palynofacies' analysis, has classically been used to determine the petrographic composition of particulate OM (Tyson, 1995). The purposes of the current work were to (1) study the spatial distribution of the principal sources of organic matter in a present-day wetland (i.e. Vernier Marsh), (2) define the optical markers of both the sources and depositional environments of $O M$ and (3) use these markers to reconstruct paleoenvironments of Holocene fluvio-palustrine deposits (i.e. Seine Valley filling).

\section{Area descriptions and material studied}

\subsection{Site description}

The Vernier Marsh, located in an abandoned meander of the Seine River (France, $49^{\circ} 25^{\prime} \mathrm{N}, 0^{\circ} 27^{\prime} \mathrm{E}$ ), is the largest remaining wetland system in the region (about 4500 ha; Fig. 1). The bedrock is Upper Cretaceous chalk and the surface of the marsh ranges between altitudes of 2 or $3 \mathrm{~m}$ above sea level. The northern part harbours an alluvial marsh and a polder, while the southern part shelters a peaty marsh (Fig. 1c). This peaty zone (about 1600 ha) presents a mosaic of environments, which differ according to the nature of water resources, the origin and nature of the sedimentary particle supply, and the local ecological conditions (Mesnage et al., 2002). In the southwestern part, terrestrial environments are composed of wet meadows and wooded parcels, and cultivated and grazed fields. Cattails (Typha) and reeds (Phragmites) characterize the vegetation of the wettest areas. In slightly drier wet meadows, rushes (J uncus), sedges (Carex) and various grasses 
(Poaceae) are dominant. The hills surrounding the marsh on three sides are covered by oaks (Querc us) and beech (Fagus). The eastern part of the peaty marsh is occupied by a pond (the Grand-Mare) in which both drainage ditches and foot slope springs are empty (Fig. 1c). The water surface covers an area of about $40 \mathrm{ha}$, with a mean depth of $0.5 \mathrm{~m}$. Before 1995, this pond has been periodically fed with Seine River inputs via the Saint-Aubin Channel. A lock that is regularly opened to manage the pond water level during the dry season controls flow in the channel. Several shallow and closed pools are associated with the Grand-Mare. The two largest ones are the Crevasse pool, which collects drainage ditch water, and the Petite-Mare pool, which is isolated from the Grand-Mare pond (Fig. 1d). 


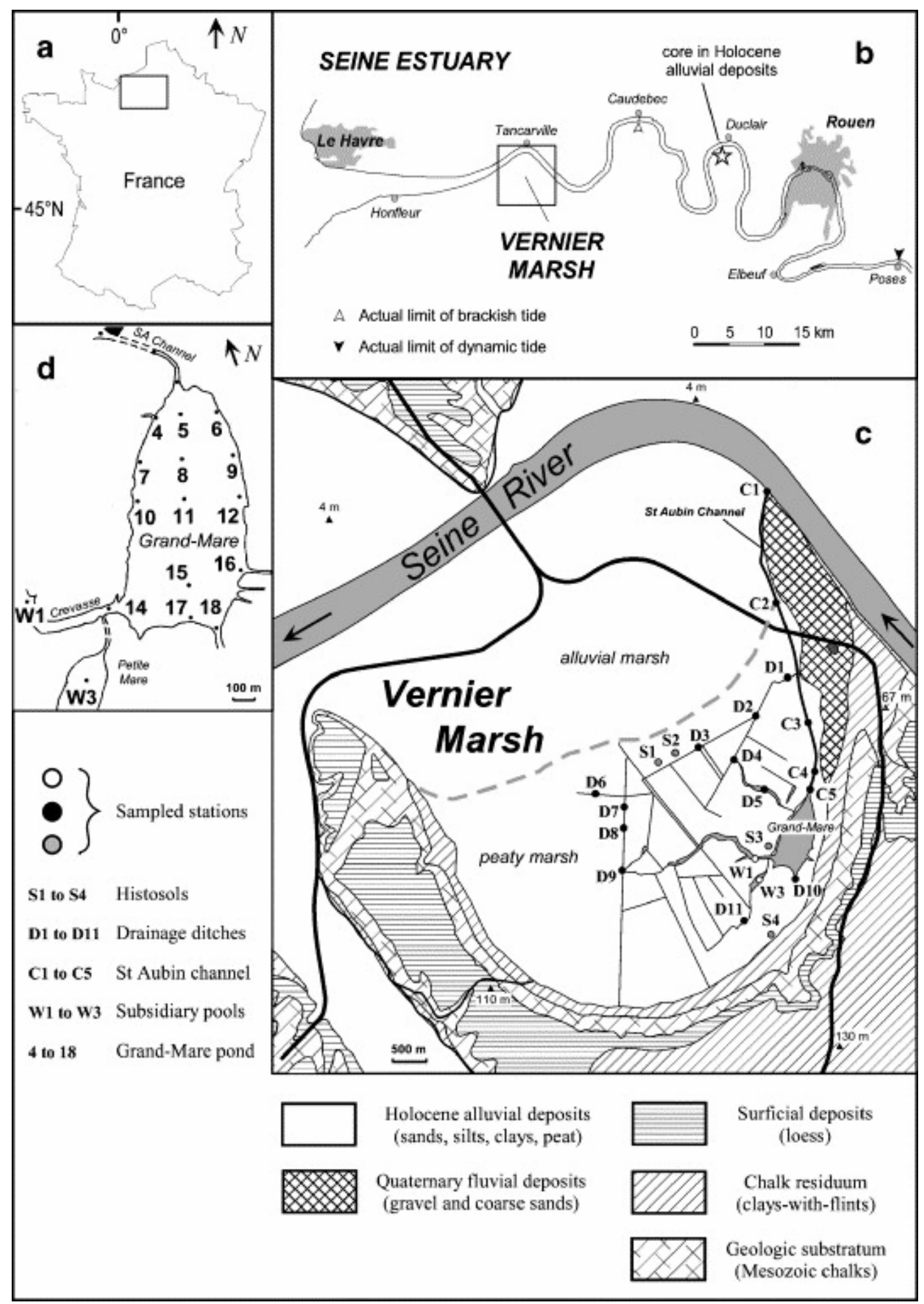

Fig. 1. Location of study site and sampling stations. (a) Location of the Lower Seine Valley in France. (b) Details of the Lower Seine Valley. (c) Simplified geomorphologic map of the Vernier Marsh and location of terrestrial, ditch and channel sampling stations. (d) Location of sampling stations in the Grand-Mare and others ponds. 


\subsection{Sampling}

Surface sediments were sampled at 40 stations in aquatic (33 stations) and terrestrial (7 stations) areas (Fig. 1; Table 1). Several environments were sampled according to their function in the depositional system (source areas, transportation systems, sites of deposition). Histosols (4 stations, 10 samples) are representative of wet terrestrial environments (Fig. 1c). On profile, two horizons were distinguished in histosols according to the abundance of fresh plant tissues, these were plant litter horizons $(\mathrm{L})$ and humic layers $(\mathrm{H})$. Surficial sediments in ditches (10 to $20 \mathrm{~cm}$ depth) were sampled at various points in the wetland (10 stations; Fig. 1c). Surficial sediments (10 to $20 \mathrm{~cm}$ depth) were also sampled in shallow open water environments (20 stations), as spot samples in pools and according to a more or less regular grid in the GrandMare (Fig. 1C). Recent alluvial deposits were sampled on the bank of the Seine River and are considered to characterize materials of fluvial origin (3 samples; not plotted). In addition, several forest profiles were sampled in the catchment area (3 stations, 8 samples; not plotted). For those, four principal horizons were distinguished according to the state of degradation of OM and the abundance of mineral materials. These horizons are identified at plant litter (L), fragmentation (F), and humic (H) layers (Table 1).

Holocene alluvial deposits constitute a representative sequence of Holocene sediments in the Seine Estuary (Fig. 1b). In the fluvial part of the Seine estuary, the sedimentary fill includes clayey and sandy loams and a thick organic sequence $(4$ to $6 \mathrm{~m})$. These peaty deposits represent the existence of a large wetland system between $5300-5000$ years BC and $1000-750$ years BC. Detailed sedimentological and paleobiological studies have already been carried out on the core; sixty-three samples were studied using palynofacies' analysis (Sebag, 2002).

\section{Palynofacies analysis}

Palynofacies' analysis consists of optical analysis of organic materials that have been isolated and concentrated from carbonate and silicate phases of the sediment (i.e. kerogen, in Combaz, 1964). The digestion of mineral materials consists of hydrochloric $(36 \%, 30 \mathrm{~min})$ and hydrofluoric $(40 \%, 45 \mathrm{~min})$ attacks. These treatments lead to the loss of some organic compounds, but previous works have shown that this acid-soluble fraction represents less than $5 \%$ of total OM in geological formations and recent sedimentary deposits, and less than 10\% of the soil OM (in Di Giovanni et al., 1999a and Di Giovanni et al., 1999b). Often performed in conjunction with bulk geochemical analyses (Meyers, 1997), it is a means of determining the identity and quantity of various organic sedimentary constituents (Tyson, 1995). 
Table 1. : Palynofacies analysis and optical indices of surficial wetland deposits and soil profiles

\begin{tabular}{|c|c|c|c|c|c|c|c|c|c|c|c|c|c|c|c|c|}
\hline \multicolumn{3}{|c|}{ Samples } & \multicolumn{6}{|c|}{$\begin{array}{l}\text { Particulate organic } \\
\text { abundances, rounded) }\end{array}$} & \multicolumn{4}{|c|}{ matter (relative } & \multicolumn{4}{|c|}{ Optical indices } \\
\hline $\begin{array}{l}\text { Locat } \\
\text { ion }\end{array}$ & $\begin{array}{l}\text { Ind } \\
\text { ex }\end{array}$ & $\begin{array}{l}\text { Typ } \\
\text { e }\end{array}$ & $\begin{array}{l}\text { A } \\
\mathbf{O} \\
\text { M } \\
(\% \\
)^{2}\end{array}$ & $\begin{array}{l}\text { C } \\
\text { M } \\
\text { ( } \\
\% \\
1\end{array}$ & $\begin{array}{l}\text { Th } \\
\text { C } \\
\text { ( } \\
\% \\
1\end{array}$ & $\begin{array}{l}\text { AL } \\
\text { C } \\
\text { ( } \\
\% \\
\text { ) }\end{array}$ & $\begin{array}{l}\text { A } \\
\mathbf{P} \\
( \\
\% \\
)\end{array}$ & $\begin{array}{l}\text { G } \\
\mathbf{P} \\
( \\
\% \\
\text { ) }\end{array}$ & $\begin{array}{l}\mathbf{O} \\
\mathbf{P} \\
\text { ( } \\
\% \\
\text { ) }\end{array}$ & $\begin{array}{l}M \\
\text { YC } \\
(\% \\
)\end{array}$ & $\begin{array}{l}20 \\
0 \\
(\% \\
)\end{array}$ & $\begin{array}{l}\mathbf{S} \\
\mathbf{P} \\
\mathbf{1} \\
\% \\
1\end{array}$ & $\begin{array}{l}\text { A } \\
0 \\
M \\
(\% \\
)^{*}\end{array}$ & $\begin{array}{l}\text { Presen } \\
\text { ed/ } \\
\text { transfor } \\
\text { med }\end{array}$ & $\begin{array}{l}\text { Gelifie } \\
\text { d/ } \\
\text { amorp } \\
\text { hous }\end{array}$ & $\begin{array}{l}\mathbf{O} \\
\mathbf{P} \\
(\% \\
)^{*}\end{array}$ \\
\hline \multirow[t]{5}{*}{$\begin{array}{l}\text { Chal } \\
\text { k } \\
\text { plate } \\
\text { au }\end{array}$} & $L$ & $\begin{array}{l}\text { Fore } \\
\text { st } \\
\text { soil } \\
\text { (L) }\end{array}$ & 5 & $\begin{array}{l}1 \\
0\end{array}$ & $\begin{array}{l}1 \\
5\end{array}$ & 20 & $\begin{array}{l}2 \\
0\end{array}$ & $\begin{array}{l}1 \\
0\end{array}$ & 5 & 15 & 0 & $\begin{array}{l}< \\
5\end{array}$ & 5 & 1.26 & 0.41 & 7 \\
\hline & $\mathrm{F}$ & $\begin{array}{l}\text { Fore } \\
\text { st } \\
\text { soil } \\
\text { (F) }\end{array}$ & 10 & $\begin{array}{l}1 \\
0\end{array}$ & $\begin{array}{l}< \\
5\end{array}$ & 10 & $\begin{array}{l}2 \\
5\end{array}$ & $\begin{array}{l}1 \\
0\end{array}$ & 5 & 15 & 0 & 0 & 12 & 0.55 & 0.37 & 7 \\
\hline & $\mathrm{H}$ & $\begin{array}{l}\text { Fore } \\
\text { st } \\
\text { soil } \\
\text { (H) }\end{array}$ & 15 & 5 & tr. & 10 & $\begin{array}{l}2 \\
5\end{array}$ & $\begin{array}{l}1 \\
0\end{array}$ & $\begin{array}{l}1 \\
0\end{array}$ & 5 & 0 & 0 & 16 & 0.41 & 0.46 & 11 \\
\hline & L & $\begin{array}{l}\text { Fore } \\
\text { st } \\
\text { soil } \\
\text { (L) }\end{array}$ & tr. & $\begin{array}{l}1 \\
0\end{array}$ & $\begin{array}{l}2 \\
0\end{array}$ & 30 & $\begin{array}{l}2 \\
0\end{array}$ & $\begin{array}{l}1 \\
5\end{array}$ & 5 & 5 & 0 & 0 & 1 & 1.29 & 0.62 & 7 \\
\hline & $\mathrm{F}$ & $\begin{array}{l}\text { Fore } \\
\text { st } \\
\text { soil } \\
\text { (F) }\end{array}$ & 10 & 5 & $\begin{array}{l}1 \\
0\end{array}$ & 15 & $\begin{array}{l}2 \\
5\end{array}$ & $\begin{array}{l}1 \\
5\end{array}$ & 5 & 15 & 0 & tr. & 8 & 0.68 & 0.50 & 5 \\
\hline \multirow[t]{3}{*}{$\begin{array}{l}\text { Seine } \\
\text { valle } \\
\text { y }\end{array}$} & $L$ & $\begin{array}{l}\text { Fore } \\
\text { st } \\
\text { soil } \\
\text { (L) }\end{array}$ & tr. & $\begin{array}{l}2 \\
5\end{array}$ & $\begin{array}{l}1 \\
5\end{array}$ & 20 & 5 & $\begin{array}{l}2 \\
0\end{array}$ & $\begin{array}{l}< \\
5\end{array}$ & 15 & 0 & tr. & 1 & 2.15 & 3.80 & 3 \\
\hline & $\mathrm{F}$ & $\begin{array}{l}\text { Fore } \\
\text { st } \\
\text { soil } \\
\text { (F) }\end{array}$ & tr. & $\begin{array}{l}1 \\
5\end{array}$ & 5 & 15 & $\begin{array}{l}1 \\
5\end{array}$ & $\begin{array}{l}2 \\
5\end{array}$ & 5 & 15 & tr. & tr. & 1 & 0.77 & 1.47 & 5 \\
\hline & $\mathrm{H}$ & $\begin{array}{l}\text { Fore } \\
\text { st } \\
\text { soil } \\
\text { (H) }\end{array}$ & $<5$ & $\begin{array}{l}1 \\
0\end{array}$ & $\begin{array}{l}< \\
5\end{array}$ & 10 & $\begin{array}{l}3 \\
5\end{array}$ & $\begin{array}{l}1 \\
5\end{array}$ & $\begin{array}{l}1 \\
0\end{array}$ & 15 & 0 & $\begin{array}{l}< \\
5\end{array}$ & 2 & 0.42 & 0.45 & 11 \\
\hline $\begin{array}{l}\text { Peat } \\
\text { y } \\
\text { mars } \\
\text { h }\end{array}$ & $\begin{array}{l}\text { S1 } \\
(\mathrm{L})\end{array}$ & $\begin{array}{l}\text { Histo } \\
\text { sol } \\
\text { (5 } \\
\mathrm{cm})\end{array}$ & 10 & 5 & 5 & 15 & $\begin{array}{l}3 \\
5\end{array}$ & $\begin{array}{l}2 \\
0\end{array}$ & 5 & tr. & 0 & 5 & 11 & 0.43 & 0.59 & 4 \\
\hline
\end{tabular}




\begin{tabular}{|c|c|c|c|c|c|c|c|c|c|c|c|c|c|c|c|c|}
\hline \multicolumn{3}{|c|}{ Samples } & \multicolumn{10}{|c|}{$\begin{array}{l}\text { Particulate organic } \\
\text { abundances, rounded) }\end{array}$} & \multicolumn{4}{|c|}{ Optical indices } \\
\hline \multirow[t]{10}{*}{$\begin{array}{l}\text { Loc at } \\
\text { ion }\end{array}$} & $\begin{array}{l}\text { Ind } \\
\text { ex }\end{array}$ & $\begin{array}{l}\text { Typ } \\
\text { e }\end{array}$ & $\begin{array}{l}\mathbf{A} \\
\mathbf{O} \\
\mathbf{M} \\
(\% \\
)\end{array}$ & $\begin{array}{l}\text { C } \\
\text { M } \\
\text { ( } \\
\% \\
\text { ) }\end{array}$ & $\begin{array}{l}\text { TL } \\
\text { C } \\
\text { ( } \\
\% \\
\text { ) }\end{array}$ & $\begin{array}{l}\text { AL } \\
\text { C } \\
\text { ( } \\
\% \\
\text { ) }\end{array}$ & $\begin{array}{l}\text { A } \\
\mathbf{P} \\
\text { ( } \\
\% \\
\text { ) }\end{array}$ & $\begin{array}{l}\mathbf{G} \\
\mathbf{P} \\
\mathbf{l} \\
\% \\
\mathbf{0}\end{array}$ & $\begin{array}{l}\mathbf{0} \\
\mathbf{P} \\
\text { ( } \\
\% \\
\text { ) }\end{array}$ & $\begin{array}{l}M \\
Y C \\
(\% \\
)\end{array}$ & $\begin{array}{l}20 \\
0 \\
(\% \\
)\end{array}$ & $\begin{array}{l}\mathbf{S} \\
\mathbf{P} \\
\mathbf{1} \\
\% \\
1\end{array}$ & $\begin{array}{l}\text { A } \\
\mathbf{0} \\
\text { M } \\
(\% \\
)^{*}\end{array}$ & $\begin{array}{l}\text { Presen } \\
\text { ed/ } \\
\text { transfor } \\
\text { med }\end{array}$ & $\begin{array}{l}\text { Gelifie } \\
\text { d/ } \\
\text { amorp } \\
\text { hous }\end{array}$ & $\begin{array}{l}\mathbf{O} \\
\mathbf{P} \\
(\% \\
)^{*}\end{array}$ \\
\hline & $\begin{array}{l}S 1 \\
(H)\end{array}$ & $\begin{array}{l}\text { Histo } \\
\text { sol } \\
\text { (10 } \\
\mathrm{cm})\end{array}$ & 15 & 5 & 5 & 10 & $\begin{array}{l}3 \\
5\end{array}$ & $\begin{array}{l}2 \\
3\end{array}$ & 5 & 5 & 0 & 0 & 14 & 0.26 & 0.66 & 7 \\
\hline & $\begin{array}{l}\text { S2 } \\
\text { (L) }\end{array}$ & $\begin{array}{l}\text { Histo } \\
\text { sol } \\
\text { (5 } \\
\mathrm{cm})\end{array}$ & 15 & 5 & 5 & 5 & $\begin{array}{l}3 \\
0\end{array}$ & $\begin{array}{l}2 \\
0\end{array}$ & $\begin{array}{l}1 \\
0\end{array}$ & 15 & 0 & tr. & 13 & 0.29 & 0.67 & 11 \\
\hline & $\begin{array}{l}\text { S2 } \\
(H)\end{array}$ & $\begin{array}{l}\text { Histo } \\
\text { sol } \\
\text { (10 } \\
\mathrm{cm})\end{array}$ & 15 & 5 & 5 & 5 & $\begin{array}{l}3 \\
0\end{array}$ & $\begin{array}{l}2 \\
0\end{array}$ & $\begin{array}{l}2 \\
0\end{array}$ & 5 & 0 & tr. & 16 & 0.24 & 0.67 & 18 \\
\hline & $\begin{array}{l}\text { S3 } \\
\text { (L) }\end{array}$ & $\begin{array}{l}\text { Histo } \\
\text { sol } \\
\text { (5 } \\
\mathrm{cm})\end{array}$ & tr. & 5 & 5 & 5 & $\begin{array}{l}3 \\
0\end{array}$ & $\begin{array}{l}1 \\
5\end{array}$ & 5 & tr. & 0 & tr. & 1 & 0.18 & 0.26 & 4 \\
\hline & $\begin{array}{l}\text { S3 } \\
(H)\end{array}$ & $\begin{array}{l}\text { Histo } \\
\text { sol } \\
\text { (10 } \\
\mathrm{cm})\end{array}$ & 10 & tr. & tr. & 5 & $\begin{array}{l}4 \\
5\end{array}$ & $\begin{array}{l}2 \\
5\end{array}$ & 5 & tr. & 0 & tr. & 10 & 0.13 & 0.53 & 5 \\
\hline & $\begin{array}{l}\text { S3 } \\
(H)\end{array}$ & $\begin{array}{l}\text { Histo } \\
\text { sol } \\
\text { (15 } \\
\mathrm{cm})\end{array}$ & 15 & 5 & $\begin{array}{l}< \\
5\end{array}$ & 5 & $\begin{array}{l}3 \\
5\end{array}$ & $\begin{array}{l}2 \\
5\end{array}$ & $\begin{array}{l}1 \\
0\end{array}$ & $<5$ & 0 & 0 & 15 & 0.22 & 0.79 & 8 \\
\hline & $\begin{array}{l}\text { S4 } \\
(\mathrm{L})\end{array}$ & $\begin{array}{l}\text { Histo } \\
\text { sol } \\
\text { (5 } \\
\mathrm{cm})\end{array}$ & 10 & $\begin{array}{l}< \\
5\end{array}$ & 5 & 10 & $\begin{array}{l}4 \\
0\end{array}$ & $\begin{array}{l}1 \\
5\end{array}$ & 5 & 5 & 0 & tr. & 10 & 0.33 & 0.28 & 6 \\
\hline & $\begin{array}{l}\text { S4 } \\
\text { (H) }\end{array}$ & $\begin{array}{l}\text { Histo } \\
\text { sol } \\
(10 \\
\mathrm{cm})\end{array}$ & 10 & $\begin{array}{l}< \\
5\end{array}$ & 5 & 5 & $\begin{array}{l}3 \\
0\end{array}$ & $\begin{array}{l}3 \\
0\end{array}$ & $\begin{array}{l}1 \\
0\end{array}$ & tr. & 0 & tr. & 11 & 0.18 & 0.97 & 12 \\
\hline & $\begin{array}{l}\text { S4 } \\
(\mathrm{H})\end{array}$ & $\begin{array}{l}\text { Histo } \\
\text { sol } \\
\text { (15 } \\
\mathrm{cm})\end{array}$ & 15 & $\begin{array}{l}< \\
5\end{array}$ & tr. & 5 & $\begin{array}{l}3 \\
5\end{array}$ & $\begin{array}{l}2 \\
5\end{array}$ & $\begin{array}{l}1 \\
5\end{array}$ & $<5$ & 0 & 0 & 15 & 0.12 & 0.69 & 14 \\
\hline $\begin{array}{l}\text { Saint- } \\
\text { Aubi } \\
n\end{array}$ & $\begin{array}{l}\mathrm{CO} \\
1\end{array}$ & $\begin{array}{l}\text { Surfi } \\
\text { cial } \\
\text { dep }\end{array}$ & 30 & 5 & 5 & 5 & 5 & $\begin{array}{l}1 \\
0\end{array}$ & $\begin{array}{l}3 \\
0\end{array}$ & $\operatorname{tr}$. & 0 & $\begin{array}{l}< \\
5\end{array}$ & 32 & 0.29 & 1.71 & 30 \\
\hline
\end{tabular}




\begin{tabular}{|c|c|c|c|c|c|c|c|c|c|c|c|c|c|c|c|c|}
\hline \multicolumn{3}{|c|}{ Samples } & \multicolumn{10}{|c|}{$\begin{array}{l}\text { Particulate organic } \\
\text { abundances, rounded) }\end{array}$} & \multicolumn{4}{|c|}{ Optical indices } \\
\hline $\begin{array}{l}\text { Locat } \\
\text { ion }\end{array}$ & $\begin{array}{l}\text { Ind } \\
\text { ex }\end{array}$ & $\begin{array}{l}\text { Typ } \\
\text { e }\end{array}$ & $\begin{array}{l}\text { A } \\
\text { O } \\
\text { M } \\
(\% \\
)\end{array}$ & $\begin{array}{l}\text { C } \\
\text { M } \\
( \\
\% \\
\text { ) }\end{array}$ & $\begin{array}{l}\text { Th } \\
\text { C } \\
\text { ( } \\
\% \\
\text { ) }\end{array}$ & $\begin{array}{l}\text { AL } \\
\text { C } \\
\text { ( } \\
\% \\
\text { ) }\end{array}$ & $\begin{array}{l}\text { A } \\
\mathbf{P} \\
( \\
\% \\
)\end{array}$ & $\begin{array}{l}\mathbf{G} \\
\mathbf{P} \\
\mathbf{l} \\
\% \\
\mathbf{0}\end{array}$ & $\begin{array}{l}\mathbf{0} \\
\mathbf{P} \\
\text { ( } \\
\% \\
\text { ) }\end{array}$ & $\begin{array}{l}\text { M } \\
\text { YC } \\
(\% \\
)\end{array}$ & $\begin{array}{l}20 \\
0 \\
(\% \\
1\end{array}$ & $\begin{array}{l}\mathbf{S} \\
\mathbf{P} \\
\mathbf{1} \\
\% \\
1\end{array}$ & $\begin{array}{l}\text { A } \\
\mathbf{0} \\
\text { M } \\
\text { (\% } \\
)^{*}\end{array}$ & $\begin{array}{l}\text { Presen } \\
\text { ed/ } \\
\text { transfor } \\
\text { med }\end{array}$ & $\begin{array}{l}\text { Gelifie } \\
\text { d/ } \\
\text { amorp } \\
\text { hous }\end{array}$ & $\begin{array}{l}\mathbf{O} \\
\mathbf{P} \\
(\% \\
)^{*}\end{array}$ \\
\hline \multirow{4}{*}{$\begin{array}{l}\text { Chan } \\
\text { nel }\end{array}$} & & osit & & & & & & & & & & & & & & \\
\hline & $\begin{array}{l}\mathrm{CO} \\
2\end{array}$ & $\begin{array}{l}\text { Surfi } \\
\text { cial } \\
\text { dep } \\
\text { osit }\end{array}$ & 45 & $\begin{array}{l}< \\
5\end{array}$ & 5 & 5 & 5 & $\begin{array}{l}1 \\
0\end{array}$ & $\begin{array}{l}3 \\
0\end{array}$ & tr. & tr. & tr. & 46 & 0.30 & 1.40 & 28 \\
\hline & $\begin{array}{l}\mathrm{CO} \\
3\end{array}$ & $\begin{array}{l}\text { Surfi } \\
\text { cial } \\
\text { dep } \\
\text { osit }\end{array}$ & 35 & $\begin{array}{l}1 \\
0\end{array}$ & tr. & 5 & $\begin{array}{l}1 \\
0\end{array}$ & $\begin{array}{l}1 \\
0\end{array}$ & $\begin{array}{l}2 \\
5\end{array}$ & tr. & 0 & tr. & 35 & 0.41 & 0.67 & 24 \\
\hline & $\begin{array}{l}\mathrm{CO} \\
4\end{array}$ & $\begin{array}{l}\text { Surfi } \\
\text { cial } \\
\text { dep } \\
\text { osit }\end{array}$ & 40 & $\begin{array}{l}< \\
5\end{array}$ & 5 & 5 & 5 & tr. & $\begin{array}{l}3 \\
5\end{array}$ & tr. & tr. & tr. & 41 & 0.33 & 0.29 & 33 \\
\hline \multirow[t]{6}{*}{$\begin{array}{l}\text { Ditch } \\
\text { es }\end{array}$} & $\begin{array}{l}\text { DO } \\
1\end{array}$ & $\begin{array}{l}\text { Surfi } \\
\text { cial } \\
\text { dep } \\
\text { osit }\end{array}$ & 25 & $\begin{array}{l}< \\
5\end{array}$ & 5 & 5 & $\begin{array}{l}2 \\
0\end{array}$ & $\begin{array}{l}1 \\
5\end{array}$ & $\begin{array}{l}2 \\
0\end{array}$ & $<5$ & 1 & 1 & 27 & 0.33 & 0.74 & 18 \\
\hline & $\begin{array}{l}\text { DO } \\
2\end{array}$ & $\begin{array}{l}\text { Surfi } \\
\text { cial } \\
\text { dep } \\
\text { osit }\end{array}$ & 30 & 5 & $\begin{array}{l}< \\
5\end{array}$ & 5 & $\begin{array}{l}1 \\
5\end{array}$ & $\begin{array}{l}1 \\
0\end{array}$ & $\begin{array}{l}2 \\
5\end{array}$ & 5 & tr. & $\begin{array}{l}< \\
5\end{array}$ & 29 & 0.27 & 0.92 & 23 \\
\hline & $\begin{array}{l}\text { DO } \\
3\end{array}$ & $\begin{array}{l}\text { Surfi } \\
\text { cial } \\
\text { dep } \\
\text { osit }\end{array}$ & 20 & 5 & 5 & 5 & $\begin{array}{l}2 \\
0\end{array}$ & $\begin{array}{l}2 \\
0\end{array}$ & $\begin{array}{l}1 \\
5\end{array}$ & $<5$ & tr. & 2 & 22 & 0.30 & 0.82 & 16 \\
\hline & $\begin{array}{l}\text { DO } \\
4\end{array}$ & $\begin{array}{l}\text { Surfi } \\
\text { cial } \\
\text { dep } \\
\text { osit }\end{array}$ & 30 & 5 & $\begin{array}{l}< \\
5\end{array}$ & 5 & $\begin{array}{l}2 \\
0\end{array}$ & $\begin{array}{l}1 \\
5\end{array}$ & $\begin{array}{l}1 \\
0\end{array}$ & 2 & $\operatorname{tr}$. & $\begin{array}{l}< \\
5\end{array}$ & 32 & 0.32 & 0.64 & 11 \\
\hline & $\begin{array}{l}\text { D0 } \\
5\end{array}$ & $\begin{array}{l}\text { Surfi } \\
\text { cial } \\
\text { dep } \\
\text { osit }\end{array}$ & 15 & 5 & $\begin{array}{l}0 . \\
5\end{array}$ & 10 & $\begin{array}{l}4 \\
5\end{array}$ & $\begin{array}{l}1 \\
0\end{array}$ & $\begin{array}{l}1 \\
0\end{array}$ & 2 & tr. & $\begin{array}{l}< \\
5\end{array}$ & 15 & 0.25 & 0.20 & 9 \\
\hline & $\begin{array}{l}\text { D0 } \\
7\end{array}$ & $\begin{array}{l}\text { Surfi } \\
\text { cial } \\
\text { dep } \\
\text { osit }\end{array}$ & 20 & 5 & 5 & 10 & $\begin{array}{l}3 \\
5\end{array}$ & $\begin{array}{l}1 \\
0\end{array}$ & $\begin{array}{l}1 \\
0\end{array}$ & 1 & 0 & $\begin{array}{l}< \\
5\end{array}$ & 20 & 0.31 & 0.33 & 10 \\
\hline
\end{tabular}




\begin{tabular}{|c|c|c|c|c|c|c|c|c|c|c|c|c|c|c|c|c|}
\hline \multicolumn{3}{|c|}{ Samples } & \multicolumn{6}{|c|}{$\begin{array}{l}\text { Particulate organic } \\
\text { abundances, rounded) }\end{array}$} & \multicolumn{4}{|c|}{ natter (relative } & \multicolumn{4}{|c|}{ Optical indices } \\
\hline \multirow[t]{4}{*}{$\begin{array}{l}\text { Locat } \\
\text { ion }\end{array}$} & $\begin{array}{l}\text { Ind } \\
\text { ex }\end{array}$ & $\begin{array}{l}\text { Typ } \\
\text { e }\end{array}$ & $\begin{array}{l}\text { A } \\
\mathbf{O} \\
\text { M } \\
(\% \\
\text { ) }\end{array}$ & $\begin{array}{l}C \\
M \\
( \\
\% \\
)\end{array}$ & $\begin{array}{l}\text { TL } \\
\text { C } \\
\text { ( } \\
\% \\
1\end{array}$ & $\begin{array}{l}\text { AL } \\
\text { C } \\
\text { ( } \\
\% \\
)\end{array}$ & $\begin{array}{l}\text { A } \\
\mathbf{P} \\
( \\
\% \\
)\end{array}$ & $\begin{array}{l}\text { G } \\
\mathbf{P} \\
\mathbf{1} \\
\% \\
1\end{array}$ & $\begin{array}{l}\mathbf{O} \\
\mathbf{P} \\
\mathbf{1} \\
\% \\
1\end{array}$ & $\begin{array}{l}M \\
\text { YC } \\
(\% \\
)\end{array}$ & $\begin{array}{l}20 \\
0 \\
(\% \\
)\end{array}$ & $\begin{array}{l}\mathbf{S} \\
\mathbf{P} \\
\mathbf{1} \\
\% \\
1\end{array}$ & $\begin{array}{l}\text { A } \\
\mathbf{0} \\
\text { M } \\
\text { (\% } \\
\text { )* }\end{array}$ & $\begin{array}{l}\text { Presen } \\
\text { ed/ } \\
\text { transfor } \\
\text { med }\end{array}$ & $\begin{array}{l}\text { Gelifie } \\
\text { d/ } \\
\text { amorp } \\
\text { hous }\end{array}$ & $\begin{array}{l}\mathbf{O} \\
\mathbf{P} \\
(\% \\
)^{*}\end{array}$ \\
\hline & $\begin{array}{l}\text { DO } \\
9\end{array}$ & $\begin{array}{l}\text { Surfi } \\
\text { cial } \\
\text { dep } \\
\text { osit }\end{array}$ & $<5$ & $\begin{array}{l}1 \\
0\end{array}$ & $\begin{array}{l}< \\
5\end{array}$ & 15 & $\begin{array}{l}3 \\
5\end{array}$ & $\begin{array}{l}1 \\
5\end{array}$ & $\begin{array}{l}1 \\
5\end{array}$ & $<5$ & 0 & $\begin{array}{l}< \\
5\end{array}$ & 4 & 0.42 & 0.40 & 13 \\
\hline & $\begin{array}{l}\text { D1 } \\
0\end{array}$ & $\begin{array}{l}\text { Surfi } \\
\text { cial } \\
\text { dep } \\
\text { osit }\end{array}$ & 20 & 5 & $\begin{array}{l}< \\
5\end{array}$ & 10 & $\begin{array}{l}2 \\
5\end{array}$ & $\begin{array}{l}1 \\
5\end{array}$ & $\begin{array}{l}1 \\
5\end{array}$ & $<5$ & tr. & tr. & 20 & 0.40 & 0.54 & 13 \\
\hline & $\begin{array}{l}\text { D1 } \\
1\end{array}$ & $\begin{array}{l}\text { Surfi } \\
\text { cial } \\
\text { dep } \\
\text { osit }\end{array}$ & 5 & $\begin{array}{l}1 \\
0\end{array}$ & 5 & 10 & $\begin{array}{l}3 \\
0\end{array}$ & $\begin{array}{l}2 \\
0\end{array}$ & $\begin{array}{l}1 \\
0\end{array}$ & 5 & 0 & 1 & 7 & 0.42 & 0.73 & 8 \\
\hline \multirow[t]{7}{*}{$\begin{array}{l}\text { Gran } \\
\text { d- } \\
\text { Mare }\end{array}$} & $\begin{array}{l}\text { GM } \\
04\end{array}$ & $\begin{array}{l}\text { Surfi } \\
\text { cial } \\
\text { dep } \\
\text { osit }\end{array}$ & 30 & 5 & 5 & 15 & $\begin{array}{l}1 \\
5\end{array}$ & $\begin{array}{l}1 \\
5\end{array}$ & $\begin{array}{l}1 \\
0\end{array}$ & $<5$ & 0 & tr. & 28 & 0.66 & 1.13 & 9 \\
\hline & $\begin{array}{l}\text { GM } \\
05\end{array}$ & $\begin{array}{l}\text { Surfi } \\
\text { cial } \\
\text { dep } \\
\text { osit }\end{array}$ & 15 & 5 & 5 & 10 & $\begin{array}{l}2 \\
0\end{array}$ & $\begin{array}{l}2 \\
5\end{array}$ & $\begin{array}{l}1 \\
5\end{array}$ & tr. & tr. & tr. & 17 & 0.32 & 1.14 & 15 \\
\hline & $\begin{array}{l}\text { GM } \\
06\end{array}$ & $\begin{array}{l}\text { Surfi } \\
\text { cial } \\
\text { dep } \\
\text { osit }\end{array}$ & 20 & $\begin{array}{l}1 \\
0\end{array}$ & $\begin{array}{l}1 \\
0\end{array}$ & 10 & $\begin{array}{l}1 \\
5\end{array}$ & $\begin{array}{l}1 \\
5\end{array}$ & $\begin{array}{l}1 \\
5\end{array}$ & tr. & tr. & tr. & 19 & 0.60 & 0.94 & 15 \\
\hline & $\begin{array}{l}\text { GM } \\
07\end{array}$ & $\begin{array}{l}\text { Surfi } \\
\text { cial } \\
\text { dep } \\
\text { osit }\end{array}$ & 30 & $\begin{array}{l}1 \\
0\end{array}$ & 5 & 10 & $\begin{array}{l}1 \\
5\end{array}$ & $\begin{array}{l}1 \\
0\end{array}$ & $\begin{array}{l}1 \\
0\end{array}$ & tr. & tr. & tr. & 29 & 0.69 & 0.69 & 12 \\
\hline & $\begin{array}{l}\text { GM } \\
08\end{array}$ & $\begin{array}{l}\text { Surfi } \\
\text { cial } \\
\text { dep } \\
\text { osit }\end{array}$ & 35 & tr. & $\begin{array}{l}< \\
5\end{array}$ & 10 & $\begin{array}{l}1 \\
5\end{array}$ & $\begin{array}{l}2 \\
0\end{array}$ & $\begin{array}{l}1 \\
5\end{array}$ & tr. & 0 & tr. & 35 & 0.32 & 1.36 & 14 \\
\hline & $\begin{array}{l}\text { GM } \\
09\end{array}$ & $\begin{array}{l}\text { Surfi } \\
\text { cial } \\
\text { dep } \\
\text { osit }\end{array}$ & 15 & 5 & $\begin{array}{l}1 \\
0\end{array}$ & 15 & $\begin{array}{l}2 \\
0\end{array}$ & $\begin{array}{l}1 \\
5\end{array}$ & $\begin{array}{l}1 \\
5\end{array}$ & tr. & 0 & tr. & 15 & 0.61 & 0.75 & 16 \\
\hline & $\begin{array}{l}\text { GM } \\
10\end{array}$ & $\begin{array}{l}\text { Surfi } \\
\text { cial } \\
\text { dep }\end{array}$ & 30 & $\begin{array}{l}1 \\
0\end{array}$ & 5 & 10 & $\begin{array}{l}1 \\
0\end{array}$ & $\begin{array}{l}2 \\
0\end{array}$ & $\begin{array}{l}1 \\
0\end{array}$ & tr. & tr. & $\begin{array}{l}< \\
5\end{array}$ & 29 & 0.55 & 2.44 & 11 \\
\hline
\end{tabular}




\begin{tabular}{|c|c|c|c|c|c|c|c|c|c|c|c|c|c|c|c|c|}
\hline \multicolumn{3}{|c|}{ Samples } & \multicolumn{10}{|c|}{$\begin{array}{l}\text { Particulate organic } \\
\text { abundances, rounded) }\end{array}$} & \multicolumn{4}{|c|}{ Optical indices } \\
\hline $\begin{array}{l}\text { Locat } \\
\text { ion }\end{array}$ & $\begin{array}{l}\text { Ind } \\
\text { ex }\end{array}$ & $\begin{array}{l}\text { Typ } \\
\text { e }\end{array}$ & $\begin{array}{l}\mathbf{A} \\
\mathbf{O} \\
\mathbf{M} \\
(\% \\
)\end{array}$ & $\begin{array}{l}\text { C } \\
\text { M } \\
( \\
\% \\
)\end{array}$ & $\begin{array}{l}\text { Th } \\
\text { C } \\
\text { ( } \\
\% \\
1\end{array}$ & $\begin{array}{l}\text { AL } \\
\text { C } \\
\text { ( } \\
\% \\
\text { ) }\end{array}$ & $\begin{array}{l}\text { A } \\
\text { P } \\
( \\
\% \\
)\end{array}$ & $\begin{array}{l}\mathbf{G} \\
\mathbf{P} \\
\mathbf{l} \\
\% \\
\mathbf{0}\end{array}$ & $\begin{array}{l}\mathbf{0} \\
\mathbf{P} \\
\text { ( } \\
\% \\
\text { ) }\end{array}$ & $\begin{array}{l}M \\
Y C \\
(\% \\
)\end{array}$ & $\begin{array}{l}20 \\
0 \\
(\% \\
1\end{array}$ & $\begin{array}{l}\mathbf{S} \\
\mathbf{P} \\
\mathbf{1} \\
\% \\
1\end{array}$ & $\begin{array}{l}\text { A } \\
\mathbf{0} \\
\text { M } \\
(\% \\
)^{*}\end{array}$ & $\begin{array}{l}\text { Presen } \\
\text { ed/ } \\
\text { transfor } \\
\text { med }\end{array}$ & $\begin{array}{l}\text { Gelifie } \\
\text { d/ } \\
\text { amorp } \\
\text { hous }\end{array}$ & $\begin{array}{l}\mathbf{O} \\
\mathbf{P} \\
(\% \\
)^{*}\end{array}$ \\
\hline & & osit & & & & & & & & & & & & & & \\
\hline & $\begin{array}{l}\text { GM } \\
11\end{array}$ & $\begin{array}{l}\text { Surfi } \\
\text { cial } \\
\text { dep } \\
\text { osit }\end{array}$ & 40 & $\begin{array}{l}< \\
5\end{array}$ & $\begin{array}{l}< \\
5\end{array}$ & 5 & 5 & $\begin{array}{l}2 \\
0\end{array}$ & $\begin{array}{l}1 \\
5\end{array}$ & tr. & 0 & tr. & 42 & 0.35 & 4.40 & 13 \\
\hline & $\begin{array}{l}\text { GM } \\
12\end{array}$ & $\begin{array}{l}\text { Surfi } \\
\text { cial } \\
\text { dep } \\
\text { osit }\end{array}$ & 15 & $\begin{array}{l}1 \\
0\end{array}$ & 5 & 15 & $\begin{array}{l}2 \\
0\end{array}$ & $\begin{array}{l}2 \\
0\end{array}$ & $\begin{array}{l}1 \\
0\end{array}$ & tr. & tr. & tr. & 14 & 0.69 & 1.17 & 9 \\
\hline & $\begin{array}{l}\text { GM } \\
14\end{array}$ & $\begin{array}{l}\text { Surfi } \\
\text { cial } \\
\text { dep } \\
\text { osit }\end{array}$ & 25 & 5 & tr. & 5 & $\begin{array}{l}1 \\
0\end{array}$ & $\begin{array}{l}3 \\
0\end{array}$ & $\begin{array}{l}1 \\
5\end{array}$ & tr. & $\begin{array}{l}< \\
5\end{array}$ & tr. & 27 & 0.26 & 2.90 & 14 \\
\hline & $\begin{array}{l}\text { GM } \\
15\end{array}$ & $\begin{array}{l}\text { Surfi } \\
\text { cial } \\
\text { dep } \\
\text { osit }\end{array}$ & 30 & 5 & 5 & 15 & $\begin{array}{l}1 \\
0\end{array}$ & $\begin{array}{l}2 \\
0\end{array}$ & $\begin{array}{l}1 \\
0\end{array}$ & tr. & $\begin{array}{l}< \\
5\end{array}$ & $\operatorname{tr}$. & 29 & 0.55 & 2.20 & 10 \\
\hline & $\begin{array}{l}\text { GM } \\
16\end{array}$ & $\begin{array}{l}\text { Surfi } \\
\text { cial } \\
\text { dep } \\
\text { osit }\end{array}$ & 30 & $\begin{array}{l}1 \\
0\end{array}$ & 5 & 10 & $\begin{array}{l}1 \\
0\end{array}$ & $\begin{array}{l}2 \\
0\end{array}$ & $\begin{array}{l}1 \\
5\end{array}$ & $<5$ & tr. & tr. & 29 & 0.49 & 2.00 & 13 \\
\hline & $\begin{array}{l}\text { GM } \\
17\end{array}$ & $\begin{array}{l}\text { Surfi } \\
\text { cial } \\
\text { dep } \\
\text { osit }\end{array}$ & 20 & $\begin{array}{l}1 \\
5\end{array}$ & $\begin{array}{l}1 \\
0\end{array}$ & 15 & $\begin{array}{l}1 \\
0\end{array}$ & $\begin{array}{l}2 \\
0\end{array}$ & $\begin{array}{l}1 \\
0\end{array}$ & $<5$ & tr. & tr. & 22 & 0.89 & 2.00 & 8 \\
\hline & $\begin{array}{l}\text { GM } \\
18\end{array}$ & $\begin{array}{l}\text { Surfi } \\
\text { cial } \\
\text { dep } \\
\text { osit }\end{array}$ & 25 & 5 & 5 & 10 & 5 & $\begin{array}{l}3 \\
0\end{array}$ & $\begin{array}{l}1 \\
0\end{array}$ & tr. & tr. & tr. & 27 & 0.53 & 4.14 & 9 \\
\hline $\begin{array}{l}\text { Crev } \\
\text { asse }\end{array}$ & WI & $\begin{array}{l}\text { Surfi } \\
\text { cial } \\
\text { dep } \\
\text { osit }\end{array}$ & 15 & 5 & 5 & 15 & $\begin{array}{l}1 \\
5\end{array}$ & $\begin{array}{l}3 \\
0\end{array}$ & $\begin{array}{l}1 \\
0\end{array}$ & $\operatorname{tr}$. & tr. & tr. & 17 & 0.41 & 1.76 & 9 \\
\hline $\begin{array}{l}\text { Petite } \\
- \\
\text { Mare }\end{array}$ & W3 & $\begin{array}{l}\text { Surfi } \\
\text { cial } \\
\text { dep } \\
\text { osit }\end{array}$ & 20 & 5 & $\begin{array}{l}< \\
5\end{array}$ & 15 & $\begin{array}{l}1 \\
5\end{array}$ & $\begin{array}{l}3 \\
0\end{array}$ & $\begin{array}{l}1 \\
0\end{array}$ & $<5$ & 0 & 0 & 20 & 0.38 & 2.00 & 8 \\
\hline
\end{tabular}


$\left({ }^{*}\right)$ : results obtained by counting. AOM: amorphous organic matter; CM: cuticles; TLC: transparent ligno-cellulosic fragments; ALC: altered ligno-cellulosic fragments; AP: amorphous particles; GP: gelified particles; OP: opaque particles; MYC: mycelium; ZOO: zooclasts; SP: spores and pollen.

This approach has been classically used to study marine geological formations, as it allows one to estimate the petroleum potential of a rock, to determine biostratigraphic correlations, to identify the principal sources of $\mathrm{OM}$, and to reconstruct the evolution of depositional environments (Batten, 1996 and Tyson, 1995). In continental deposits, palynofacies' analysis allows one to identify and distinguish the results of lacustrine (Di Giovanni et al., 2000) and palustrine processes (Laggoun-Défarge et al., 1999). It may also be used to study the effects of synsedimentary processes and early diagenetic changes in peatland deposits (Bourdon et al., 2000) or the incorporation of OM into soils (Di Giovanni et al., 1999C).

In palynofacies' preparations, several categories of particulate OM can be distinguished according to their optical properties: there include amorphous OM, discrete elements, and phytoclasts (Fig. 2a; Batten, 1996 and Tyson, 1995). In addition to these organic constituents, opaque mineral particles (e.g. sulphides, oxides) often appear in residues. 

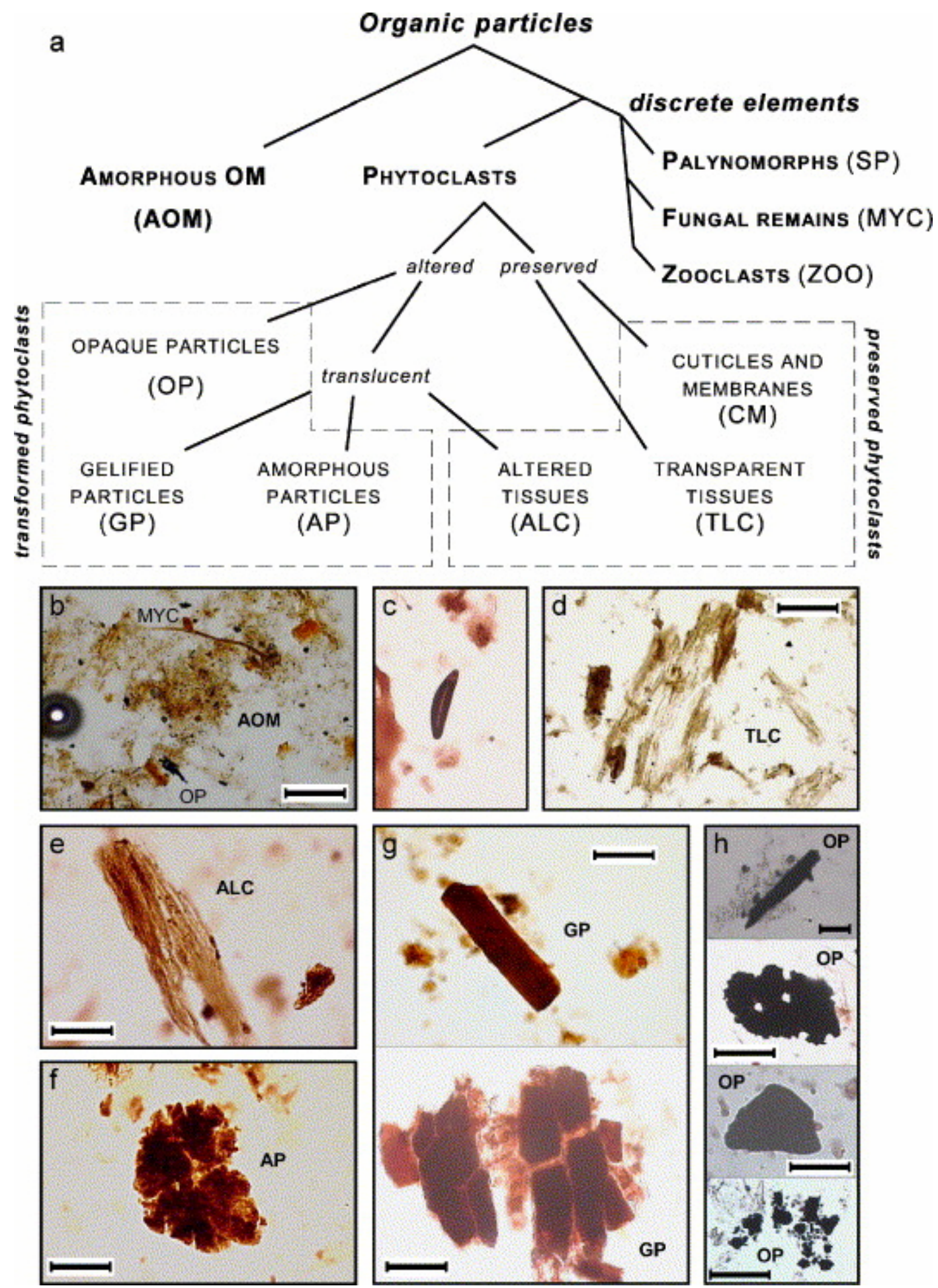

Fig. 2. (a) Simplified classification of the OM observed in transmitted optical microscope (modified from Iyson, 1995). Morphological criteria allow us to distinguish three main categories: AOM (diffuse outlines), discrete elements (recognizable organisms or organs) and phytoclasts (resulting from degradation of the plant fragments). Phytoclasts are divided into several classes according to their nature and to their degree of degradation (preserved phytoclasts) or to characters acquired during their degradation (transformed phytoclatsts), (b) amorphous organic matter (AOM), (c) discrete elements (diatom), (d) ligno-cellulosic transparent fragments (TLC), (e) reddish altered phytoclast (ALC), (f) reddish amorphous particle (AP), (g) gelified particles (GP), (h) various opaque particles (OP), scale bar: about 10 $\mu \mathrm{m}$. 


\subsection{Amorphous OM}

As seen in optical microscope (transmitted light), amorphous OM (AOM) consist of particles that are flaky, lumpy, or granular, and that have indistinct outlines. They are also typically of variable size, degree of opacity and color (Fig. 2b). AOM retains various optical, chemical and structural properties that originate as bacterial, algal or planktonic matter (Tyson, 1995).

\subsection{Discrete elements}

Discrete elements are recognizable organs or organisms (Fig. 2c). They are classified according to their taxonomic level and/or to their morphotype, these include palynomorphs (spores and pollens), fungal remains (mycelium fragments) and zooclasts (chitinous fragments of arthropods).

\subsection{Phytoc lasts}

Phytoclasts represent whole particulate constituents produced by higher plants or derived from their degradation (Fig. 2d-h). Two categories of phytoclasts were distinguished in this study: preserved phytoclasts (CM, TLC, $\mathrm{ALC}$ ) in which initial biological structures are preserved, and transformed phytoclasts (AP, GP, OP) that are characterized by acquired degradation properties. These acquired optical properties (shape, texture, opacity) depend essentially on the nature and conditions of their degradation or thermal maturation (Batten, 1996 and Tyson, 1995).

\subsubsection{Presenved phytoc lasts}

Cuticles and membranes (CM) present a fine pellicular shape and are generally of large size (> $100 \mu \mathrm{m})$. Ligno-cellulosic fragments are observed in various degradation stages. The transluscent fragments (TLC) present wellpreserved biological internal structures (Fig. 2d). Reddish altered fragments (ALC) present dull or indistinct outlines, reddish color and more or less altered internal structures (Fig. 2e).

\subsubsection{Transformed phytoc lasts}

Reddish amorphous particles (AP) appear as reddish flakes with diffuse but recognizable outlines that, sometimes, have residual internal structures (Fig. 2f). Gelified particles (GP) present a homogeneous texture, variable color (brown to amber), true outlines, and commonly angular shape but, sometimes, dulled angles (Fig. 2g). Opaque particles (OP) include various constituents which are not easily distinguished in transmitted light: the main optical criteria used to distinguish them are general shape, outline aspects and possible residual botanical structures (Fig. 2h). 
The quantitative palynofacies' composition is determined by counting particles, as they are seen in the microscope. The use of an objective with a reticule network allows the viewer to assign a value to each particle that is proportional to its apparent surface area. The results are, thus, expressed as relative areal percentages that are rounded to the closest $5 \%$. To guarantee measurement reproducibility, we analysed each slide until (1) at least 15 optical areas have been studied and 300 particles were counted in order to consider the distribution of particles on the slide, and (2) the variations of relative abundances among the last five areas were lower than $5 \%$ in order to reduce the influence of the largest particles.

\section{Results}

\subsection{Quantitative composition}

The average composition of the samples was determined from the relative abundance of the principal organic constituents (Fig. 3). Compositions consisted, on average, of $70 \%$ phytoclasts and 25\% AOM. The discrete elements (MYC, ZOO, SP) represent less than $5 \%$ of any samples. Table 1 presents the compositional assemblages of samples collected in the different parts of the depositional system, including forest soils (L, F, H) and histosols (S), channel deposits ( $C 1$ to $C 4$ ), ditches (D1 to D11), ponds (GM4 to GM18) and pools (W1 to W3).

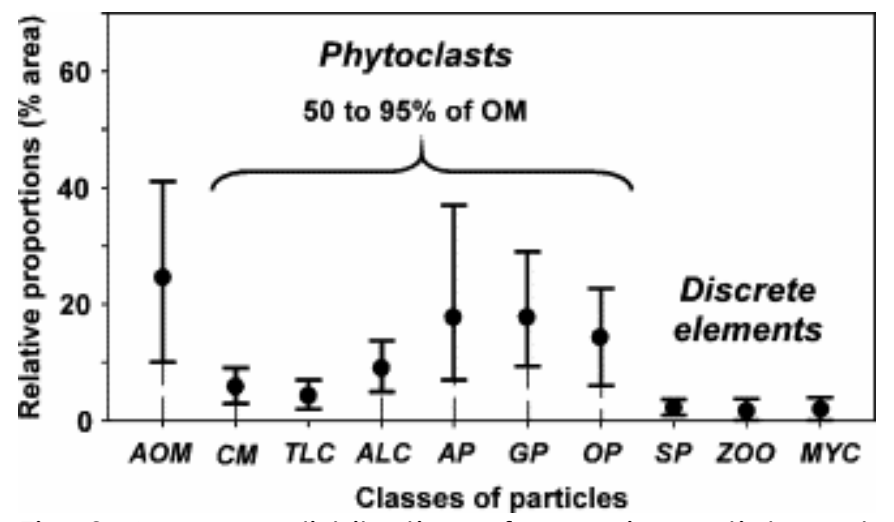

Fig. 3. Average distribution of organic particles established by palynofacies' analysis (relative areas). AOM: amorphous organic matter; CM: cuticles; TLC: transparent ligno-cellulosic fragments; ALC: altered ligno-cellulosic fragments; AP: amorphous particles; GP: gelified particles; OP: opaque particles; SP: spores and pollen; ZOO: zooclasts; MYC: mycelium.

Phytoclasts are dominant in soils (range of $75 \%$ to $90 \%$ in L horizons) and minimal in channel deposits (range of $30 \%$ to $65 \%$ in C samples; Table 1). The well-preserved phytoclasts (CM, TLC, ALC) represent generally less than $30 \%$ (except in the plant litters and some subquaeous deposits; Table 1), whereas transformed phytoclasts (AP, GP and OP) range from $35 \%$ to $60 \%$ of the OM. These latter are composed, on average, of $17 \%$ amorphous particles (AP), $16 \%$ gelified particles (GP), and 14\% opaque particles (OP). The amorphous OM (AOM) contents vary according to the sampled stations: they are less 
than $15 \%$ in soils, between $15 \%$ and $30 \%$ in the majority of drainage ditches, more than $15 \%$ in ponds, and more than $30 \%$ in Saint-Aubin Channel (Table 1). In addition, slight variations of AOM contents were measured for some stations. The sedimentary OM of modern marsh deposits includes four main fractions (AOM, AP, GP and OP) there together constitute a range from $60 \%$ to $90 \%$ of $O M$.

\subsection{Palynofacies and depositional environments}

Quantitative composition can be used to define the palynofacies' distribution of different environments of the depositional system (Fig. 4). Terrestrial environments and drainage ditches show similar palynofacies' distributions (Fig. $4 a$ and b). The major organic fractions are mainly AP (generally more than $30 \%)$ and GP (15\% to 25\%) with lesser amounts of OP and AOM (10\% to $15 \%)$. However some differences occur in ditches with a relative enrichment in AOM and a relative impoverishment in AP and GP contents compared to histosols.

Open water environments (pools and ponds) show a palynofacies distribution dominated by AOM (30\%; Fig. 4c and d). Differences between the northern and southern part of the Grand-Mare appear in phytoclast contents: AP contents are high (15\%) in the northern zone (stations 4 to 10), while GP contents are high (25\%) in the southern zone (stations 11 to 19). Finally AOM (40\%) and OP (25\%; Fig. 4e) dominate the palynofacies' distribution of the Saint-Aubin Channel.

Four palynofacies' distributions are defined in the depositional system based on the preceding data and observations: (1) AP are dominant in histosols and in drainage ditches; (2) AOM and GP are dominant in the southern zone of the Grand-Mare; (3) AOM, AP, and GP are dominant and OP contents are variable in the northern zone of the Grand-Mare; (4) AOM and OP are dominant in the Saint-Aubin Channel and in some pond and ditch stations. 

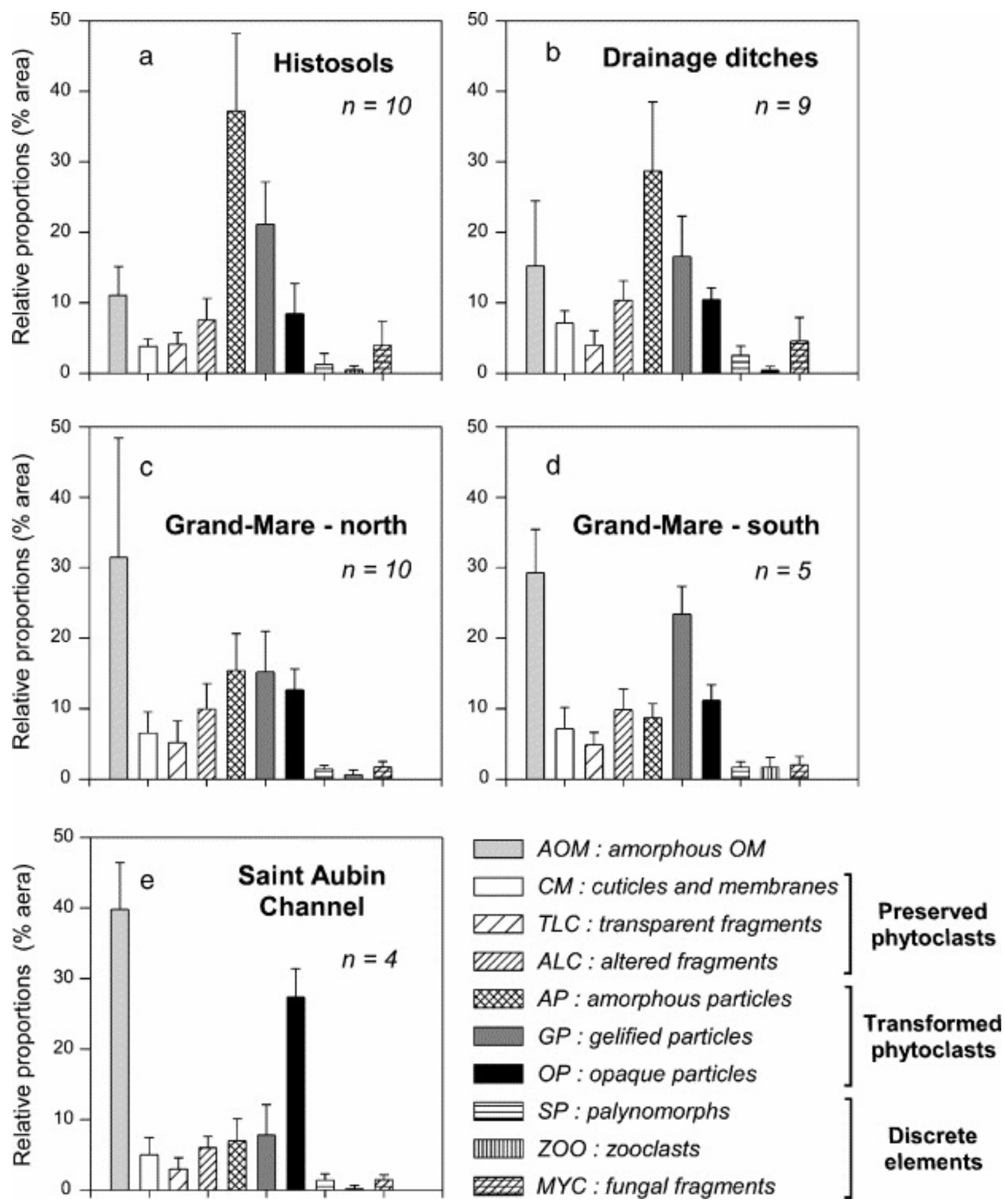

Fig. 4. Average distribution of organic particles established for each environment. The four main classes (AOM, AP, GP, OP), which are present in all environments, allow one to define four characteristic palynofacies: histosols and drainage ditches (a and b), northern (c) and southern (d) part of the Grand-Mare, and Saint-Aubin Channel (e). 


\section{Disc ussion}

\subsection{Origin of $O M$ and degradation of the temestrial fraction}

The occurrence and composition of $\mathrm{OM}$ in sediments and sedimentary rocks reflect different variables, including differences in production, transport to the depositional site, and diagenetic alterations (Tyson, 1995). We have quantified various components of the sedimentary $\mathrm{OM}$, and the results have been summarized in paired ternary diagrams. The first ternary diagram (lefthand diagrams, Fig. 5) depicts the relative proportions of $A O M$, preserved phytoclasts (CM, TLC, ALC) and transformed phytoclasts (AP, GP, OP). It is used to illustrate the origin (i.e. terrestrial or aquatic) of the principal organic particles and the state of degradation of terrestrial particles. The second ternary diagram (OP/AP/GP; right-hand diagrams, Fig. 5) relates to the early diagenesis of transformed phytoclasts. The optical properties of these particles were acquired during degradation (Batten, 1996 and Iyson, 1995). Their occurrence in all studied samples indicates, on one hand, that their genesis is not limited to a specific environment. On the other hand, these particles are not distributed uniformly in the studied palynofacies (Table 1 and Fig. 4).

\subsubsection{Terrestrial environments}

Typical soil assemblages are dominated by transformed phytoclasts (Fig. 5a). The state of preservation of phytoclasts allows one to distinguish forest litter $160 \%$ to $90 \%$ preserved tissues in L and F samples) and humic horizons (50\% to $80 \%$ transformed particle in S samples). This evolution with depth can be related to increasing AP contents (Table 1) which are a result of morphological biodegradation of plant tissues (Laggoun-Défarge et al., 1999). In agreement with previous work, these amorphous particles represent a pedogenetic OM that form in soils and peat (Bourdon et al., 2000, Di Giovanni et al., 1999C, Lallier-Vergès et al., 1998 and Noël et al., 2001) and reworked from its terrestrial source areas (Buillit et al., 1997, Patience et al., 1996 and Sifeddine et al., 1996). 
MAIN CLASSES

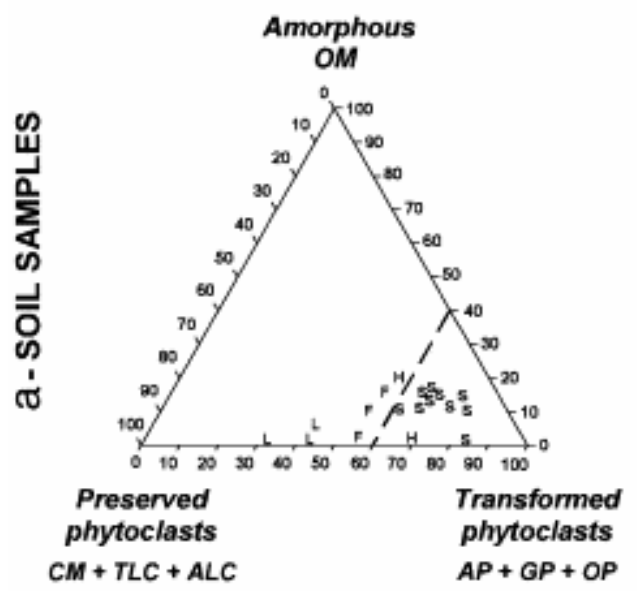

TRANSFORMED PHYTOCLASTS

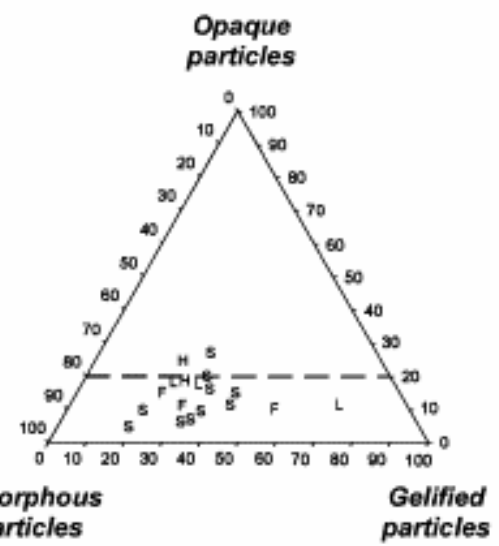

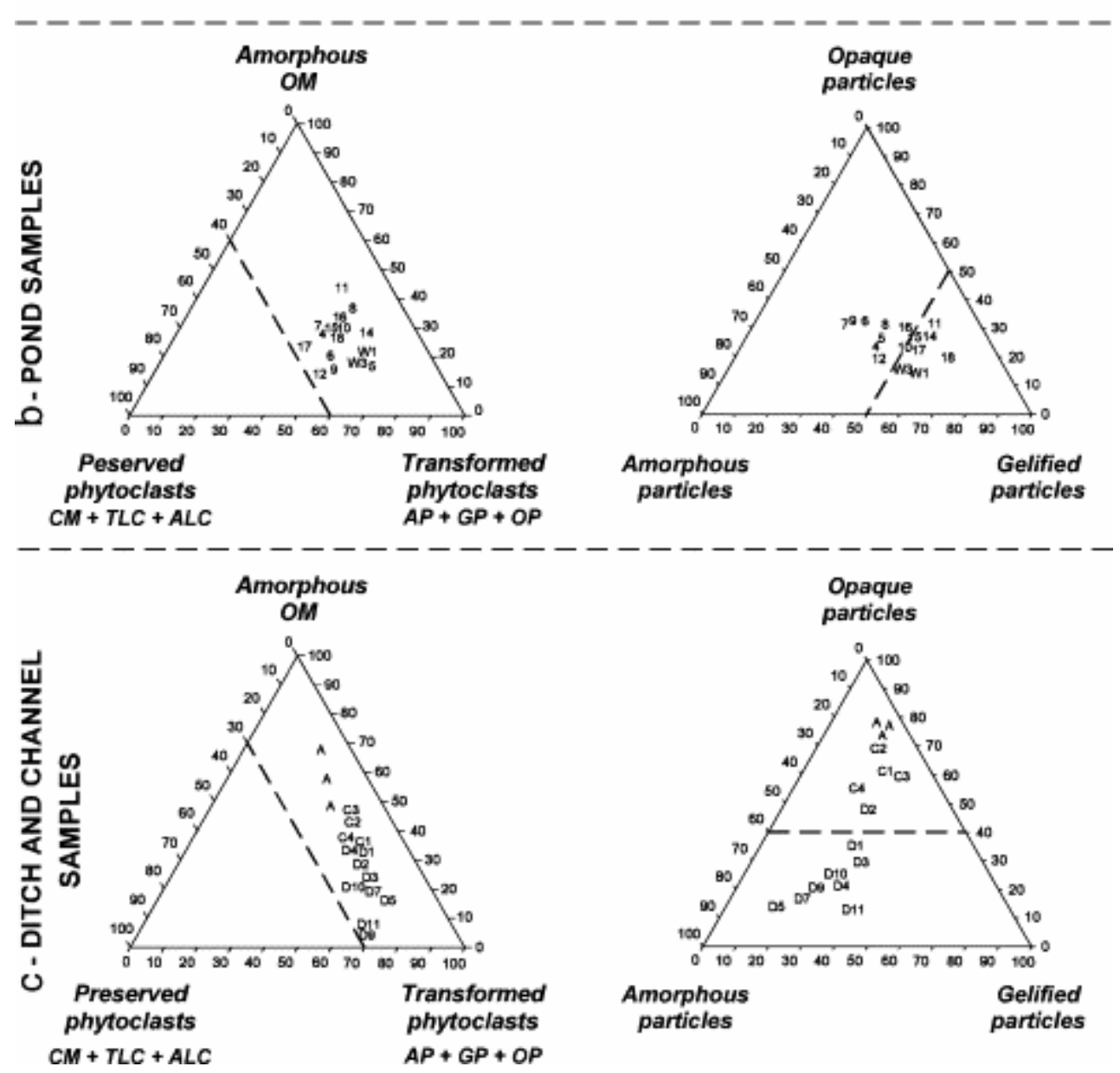

Fig. 5. Optical signatures of (a) terrestrial environments (forest soils and histosols), (b) aquatic environments (Grand-Mare pond and pools) and (C) drainage ditches and Saint-Aubin Channel. Nature of main organic constituents (AOM, preserved and transformed phytoclasts) and composition of transformed phytoclasts (amorphous, gelified or opaque particles). 


\subsubsection{Pond and pools}

In aquatic environments, $O M$ is characterized by a large variation of $A O M$ contents (10\% to $40 \%$ ). Pond samples are characterized by relatively high terrestrial plant tissues' contents (Fig. $5 \mathrm{~b}$ ). Plant fragments, which accumulate directly in aquatic environments (i.e. ponds), are less degraded than they are in aerobic environments (i.e. soils). In addition, pond environments present compositional signatures enriched in GP $(20 \%$ to $30 \%$ in the southern zone of the Grand-Mare) as compared to other environments. Previous observations (Alpern, 1976, Noël et al., 2001 and Sifeddine et al., 1998) tend to relate GP formation to the degradation of plant tissues in aquatic environments (pools, ponds, lakes) or in anaerobic conditions (catotelm of peat, hydromorphic soils). However, this point is debatable because of the variety of constituents categorized as "gelified particles" and because of the variety of factors controlling the gelification process. In spite of these uncertainties, the results presented here clearly illustrate an empirical relationship between aquatic environments and a GP enriched optical signature.

\subsubsection{Rowing water environments}

The ditch stations (D1 to D4), which receive waters from the Saint-Aubin Channel, show a relative enrichment in AOM (about $20 \%$ to $30 \%$ ) compared to other stations (D5 to D1 1). Indeed, channel deposits (C1 to C5) present an optical signature that is even more enriched with $\mathrm{AOM}(40 \%$ to $50 \%)$ than ditch deposits. In the same way, alluvial samples (A) also bear AOMdominated assemblages ( $>50 \%$ ). Thus, the range of compositions illustrates an AOM gradient from terrestrial stations (histosols, S; Fig. 5a) and soil-derived samples (ditches, D; Fig. 5c) to pond (Fig. 5b), and then fluvial deposits (A and C; Fig. $5 \mathrm{C}$ ) as a result of an increasing aquatic contribution. In flowing water environments (i.e. ditch, channel and river), the proportion of opaque particles (OP) gradually increases from drainage ditches to fluvial deposits (Fig. 5c). This change can be related to the increasing alteration of the terrestrial components during their transport (Tyson, 1995). These OP contents include heterogeneous constituents which could have various origins such as combustion residues (soot and charcoal), strongly altered and oxidized phytoclasts caused by repeated cycles of degradation (black or charcoallike phytoclasts) and/or a probable fraction degraded by thermal maturation (coalified or charred material) and inherited from the geological substratum (Di Giovanni et al., 1999b, Di Giovanni et al., 2002, Batten, 1996 and Iyson, 1995). Thus, a large portion of these opaque particles may constitute an organic fraction produced outside the system, as shown by their limited abundances in the pond and terrestrial peaty marsh environments. 


\subsection{Optical index definition}

The use of relative proportions involves semi-quantitative estimations (rounded to the nearest 5\%). The use of ratios allows one to overcome these inconveniences and to study the results statistically (i.e. using standard error and other statistical indices). In this study, the choice of four ratios is based on the preceding observations and interpretations that are summarized in the Table 2. The "AOM contents" are used to quantify the aquatic contribution. The "preserved phytoclasts / transformed phytoclasts ratio" allows one to quantify the degree of degradation of the terrestrial fraction. The "OP contents" increases with the oxidation of terrestrial source materials. The "GP / AP ratio" is used to discriminate sites which have material reworked from soil as opposed to sites where fresh plant debris fall directly into a basin, and decayed (Fig. 6). 
Table 2. : Terms and abbreviations used in this study to describe organic particles

\begin{tabular}{|c|c|c|c|c|c|}
\hline \multirow[t]{2}{*}{ Abbreviation } & \multirow[t]{2}{*}{ Designation } & \multicolumn{4}{|c|}{$\begin{array}{l}\text { Interpretations in modem depositional systems of the Lower } \\
\text { Seine Valley }\end{array}$} \\
\hline & & Origin & Nature & $\begin{array}{l}\text { Degree of } \\
\text { preservation }\end{array}$ & $\begin{array}{l}\text { Principal } \\
\text { sources } \\
\text { (conditions of } \\
\text { formation) }\end{array}$ \\
\hline$A O M$ & $\begin{array}{l}\text { Amorphous } \\
\text { organic } \\
\text { matter }\end{array}$ & Aquatic & Planktonic & $\begin{array}{l}\text { Observed in } \\
\text { various stages } \\
\text { of preservation } \\
\text { according to } \\
\text { lithology and } \\
\text { age of deposits }\end{array}$ & $\begin{array}{l}\text { No information } \\
\text { because these } \\
\text { particles are } \\
\text { related to } \\
\text { specific } \\
\text { environments }\end{array}$ \\
\hline CM & $\begin{array}{l}\text { Fragments of } \\
\text { cuticle and } \\
\text { membrane }\end{array}$ & Terrestrial & Phytoclasts & $\begin{array}{l}\text { Resistant } \\
\text { biological } \\
\text { tissues generally } \\
\text { well-preserved }\end{array}$ & $\begin{array}{l}\text { Directly from } \\
\text { vegetation, } \\
\text { plant litters }\end{array}$ \\
\hline TLC & $\begin{array}{l}\text { Transparent } \\
\text { ligno- } \\
\text { cellulosic } \\
\text { debris }\end{array}$ & Terrestrial & Phytoclasts & $\begin{array}{l}\text { Well-preserved } \\
\text { plant fragments } \\
\text { in which initial } \\
\text { biological } \\
\text { structures are } \\
\text { recognizable }\end{array}$ & $\begin{array}{l}\text { Directly from } \\
\text { vegetation, } \\
\text { fresh plant litters }\end{array}$ \\
\hline ALC & $\begin{array}{l}\text { Altered ligno- } \\
\text { cellulosic } \\
\text { debris }\end{array}$ & Terrestrial & Phytoclasts & $\begin{array}{l}\text { Transformed } \\
\text { plant debris in } \\
\text { which } \\
\text { biological } \\
\text { structures are } \\
\text { observable but } \\
\text { partially altered }\end{array}$ & $\begin{array}{l}\text { Old plant litters } \\
\text { and surficial soil } \\
\text { layers (aerobic } \\
\text { degradation of } \\
\text { ligneous plant } \\
\text { debris) }\end{array}$ \\
\hline AP & $\begin{array}{l}\text { Reddish } \\
\text { amorphous } \\
\text { particles }\end{array}$ & Terrestrial & Phytoclasts & $\begin{array}{l}\text { Degraded } \\
\text { particles } \\
\text { without } \\
\text { recognizable } \\
\text { biological } \\
\text { structures at } \\
\text { micrometer } \\
\text { scale }\end{array}$ & $\begin{array}{l}\text { Humic soil } \\
\text { layers, old plant } \\
\text { litters } \\
\text { (humification or } \\
\text { aerobic } \\
\text { diagenesis of } \\
\text { ligneous plant } \\
\text { debris) }\end{array}$ \\
\hline GP & $\begin{array}{l}\text { Gelified } \\
\text { particles }\end{array}$ & Terrestrial & Phytoclasts & $\begin{array}{l}\text { No information } \\
\text { because these } \\
\text { are particles } \\
\text { related to } \\
\text { specific } \\
\text { environments }\end{array}$ & $\begin{array}{l}\text { Hydromorphic } \\
\text { soils, catotelm } \\
\text { peat, aquatic } \\
\text { environments } \\
\text { (subaquatic } \\
\text { diagenesis of } \\
\text { plant tissues) }\end{array}$ \\
\hline OP & $\begin{array}{l}\text { Dark opaque } \\
\text { particles }\end{array}$ & Terrestrial & Phytoclasts & $\begin{array}{l}\text { Very altered } \\
\text { fragments } \\
\text { related to }\end{array}$ & $\begin{array}{l}\text { Geological } \\
\text { substratum, } \\
\text { fires, river }\end{array}$ \\
\hline
\end{tabular}




\begin{tabular}{|c|c|c|c|c|c|}
\hline \multirow[t]{2}{*}{ Abbreviation } & \multirow[t]{2}{*}{ Designation } & \multicolumn{4}{|c|}{$\begin{array}{l}\text { Interpretations in modem depositional systems of the Lower } \\
\text { Seine Valley }\end{array}$} \\
\hline & & Origin & Nature & $\begin{array}{l}\text { Degree of } \\
\text { presenvation }\end{array}$ & $\begin{array}{l}\text { Principal } \\
\text { sources } \\
\text { (conditions of } \\
\text { formation) }\end{array}$ \\
\hline & & & & $\begin{array}{l}\text { intense or } \\
\text { repeated } \\
\text { processes of } \\
\text { degradation }\end{array}$ & $\begin{array}{l}\text { transport } \\
\text { (coalification, } \\
\text { combustion, } \\
\text { oxidation) }\end{array}$ \\
\hline SP & $\begin{array}{l}\text { Spores and } \\
\text { pollens }\end{array}$ & Aerial & Palynomorphs & $\begin{array}{l}\text { Resistant } \\
\text { biological } \\
\text { products } \\
\text { generally well- } \\
\text { preserved }\end{array}$ & $\begin{array}{l}\text { Local or } \\
\text { regional } \\
\text { vegetation }\end{array}$ \\
\hline ZOO & $\begin{array}{l}\text { Organisms or } \\
\text { recognizable } \\
\text { fragments } \\
\text { (zooclasts) }\end{array}$ & Terrestrial & Zoomorphs & $\begin{array}{l}\text { Resistant } \\
\text { biological } \\
\text { remains } \\
\text { observed in } \\
\text { various stages } \\
\text { of } \\
\text { fragmentation }\end{array}$ & $\begin{array}{l}\text { Fresh plant } \\
\text { litters, acrotelm } \\
\text { peat }\end{array}$ \\
\hline MYC & $\begin{array}{l}\text { Mycelium } \\
\text { fragments }\end{array}$ & Terrestrial & Fungi & $\begin{array}{l}\text { Well-preserved } \\
\text { fragments } \\
\text { observed in } \\
\text { various stages } \\
\text { of } \\
\text { fragmentation }\end{array}$ & $\begin{array}{l}\text { Fresh plant litter, } \\
\text { acrotelm peat }\end{array}$ \\
\hline
\end{tabular}

Principal information related to their origin, nature, degree of preservation, principal source areas, and, eventually, conditions of formation. 

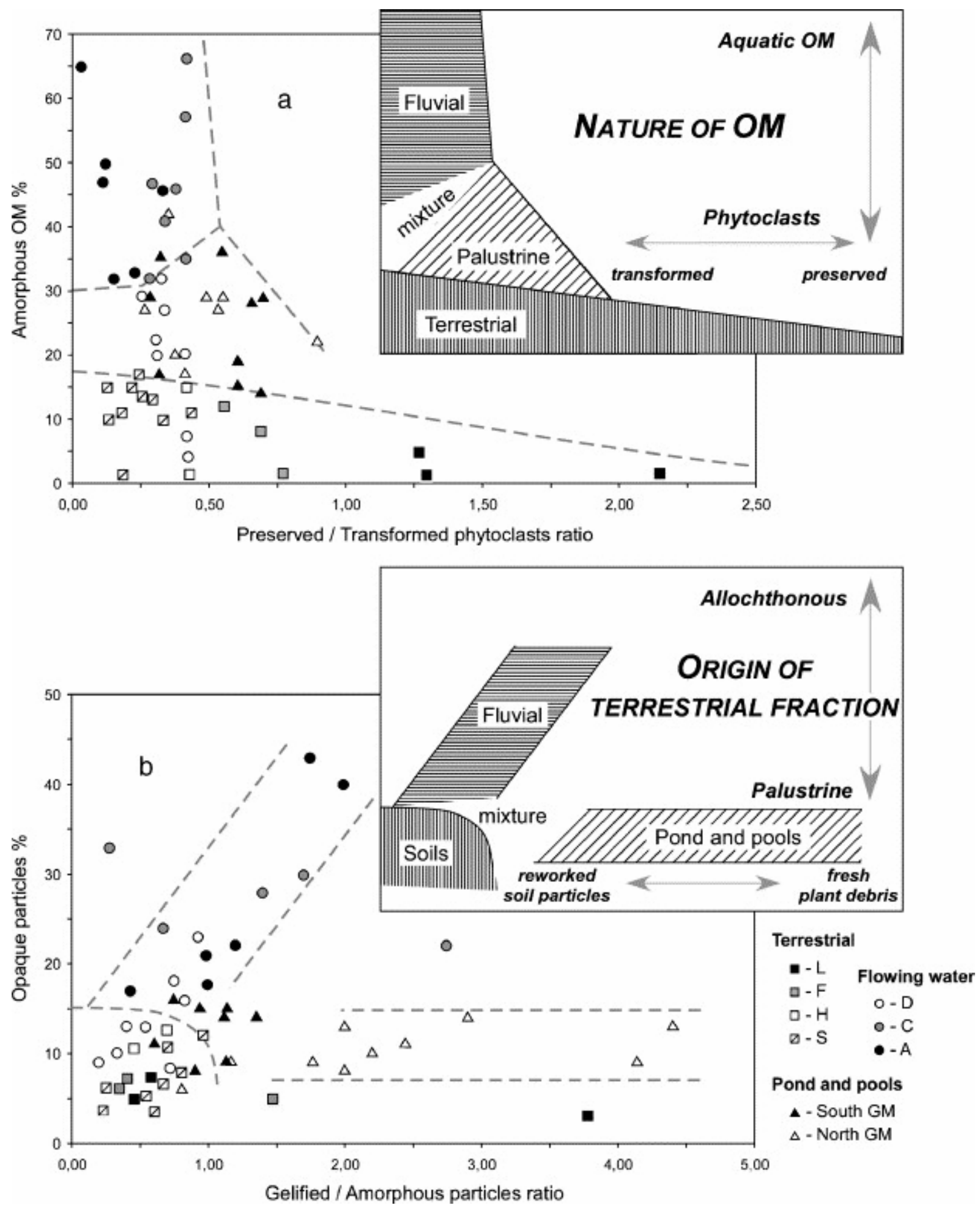

Fig. 6. Characterization of Vernier Marsh deposits with four optical indices (see text) (a) Nature of OM by using "AOM contents" (aquatic or microbial production) and "preserved / transformed phytoclasts ratio" (preservation degree of terrestrial plant debris). (b) Origin of terrestrial fraction by using "OP contents" (allochthonous fraction from fluvial origin) and "GP / AP ratio" (pedogenic or subaquatic degradation of terrestrial plant debris). Lines representing arbitrary boundaries have been plotted for guidance based on the modern environments where the samples were collected. 
Some available data from the literature support this conceptual model (Table 3). The soil profiles from various locations show the same trends as those observed in this paper. For terrestrial soil samples, the most significant index is the "preserved phytoclasts / transformed particles ratio" (Table 3). This ratio is highest in fresh litter (> 2.5), then decreases in OF horizons (to about 1.5), and has the lowest values in $\mathrm{OH}$ and $\mathrm{A}$ horizons $(<0.5)$. We also note a slight increase of OP contents with depth, there being less than $5 \%$ in the surficial layers, and up to $20 \%$ in humic layers (Table 3 ). As shown in previous work (Di Giovanni et al., 1999b), the compositional signature of organo-mineral layers (A horizons) is related to the characteristics of parent-rocks. For example, high AOM contents (> 80\%) are observed for the Draix catchment area where the parent-rock is rich in AOM (Di Giovanni, comm. pers.). In the same way, OP contents are high (>20\%) for the Chaillexon catchment area where the parent-rock is rich in OP (Di Giovanni et al., 2000), but lower (> 5\%) for Annecy catchment area where the parent-rock is poor in OP (Noël et al., 2001).

Moreover, the four new indices presented in this paper can also be used to analyse the results obtained from Holocene lacustrine (i.e. aquatic environment) or palustrine (i.e. terrestrial environment) deposits (Table 3 ). The optical signature of micritic limestones from Annecy lake corresponds precisely to an aquatic sedimentation, marked by a significant planktonic contribution (AOM contents about $40 \%$ ), and a terrestrial contribution marked by materials of detrital origin ("preserved / transformed" and "GP / AP" ratios about 0.4, "OP contents" about 5\% to 10\%). In addition, the periodic deposition of detrital sediments is recorded by a dilution of the aquatic contributions ("AOM contents" about 30\%) and an increase of detrital markers ("OP contents" about 15\%; Table 3). When these depositional events are more frequent, we note the appearance of a diagenetic fraction ("GP / AP ratio" about 1.5) derived from terrestrial sources ("preserved / transformed ratio" about 0.7). In the case of organic facies (i.e. organic mud and peaty deposits, Table 3), the AOM contents are dependent not only on the aquatic contribution $15 \%$ in palustrine deposits; $30 \%$ in lacustrine deposits from Tritrivakely marsh), but also on a possible contribution of microbial materials (about $50 \%$ in organic mud from Annecy lake). In addition, the other indices reflect the preservation state and origin of the terrestrial fraction, and the significance of the detrital inputs. 
Table 3. : Mean optical indices calculated from data of the literature

\begin{tabular}{|c|c|c|c|c|c|c|c|}
\hline \multirow[t]{2}{*}{ Location } & \multirow{2}{*}{$\begin{array}{l}\text { Sample } \\
\text { desc niption }\end{array}$} & \multirow[t]{2}{*}{$\mathbf{n}$} & \multicolumn{4}{|c|}{ Optical indices } & \multirow{2}{*}{$\begin{array}{l}\text { Referenc } \\
\text { es }\end{array}$} \\
\hline & & & $\begin{array}{l}\text { AOM } \\
\text { (\%) }\end{array}$ & $\begin{array}{l}\text { Preserved } \\
\text { / } \\
\text { transforme } \\
\text { d }\end{array}$ & $\begin{array}{l}\text { Gelified/amorpho } \\
\text { us }\end{array}$ & $\begin{array}{l}\text { OP } \\
\text { (\%) }\end{array}$ & \\
\hline \multirow{5}{*}{$\begin{array}{l}\text { Chaillexon } \\
\text { catchment } \\
\text { (Jura, } \\
\text { France) }\end{array}$} & $\begin{array}{l}\text { Recent soil } \\
\text { layers }\end{array}$ & & & & & & \\
\hline & $\begin{array}{l}\text { Fresh litters } \\
\text { (OL horizons) }\end{array}$ & 7 & $\begin{array}{l}\text { Trace } \\
\text { s }\end{array}$ & 3.1 & nd & $\begin{array}{l}\text { Trace } \\
\mathrm{s}\end{array}$ & \\
\hline & $\begin{array}{l}\text { Fragmentati } \\
\text { on layers (OF } \\
\text { horizons) }\end{array}$ & 5 & $\begin{array}{l}\text { Trace } \\
\text { s }\end{array}$ & 1.5 & nd & 6 & 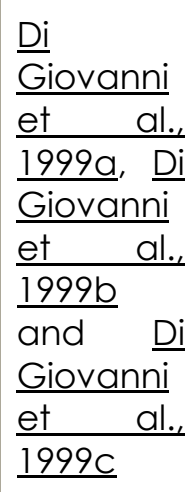 \\
\hline & $\begin{array}{l}\text { Humic layers } \\
\text { (OH } \\
\text { horizons) }\end{array}$ & 5 & $\begin{array}{l}\text { Trace } \\
\text { s }\end{array}$ & 0.5 & nd & 20 & $\begin{array}{l}\frac{\text { Di }}{\text { Giovanni }} \\
\frac{\text { et al. }}{\underline{2000}}\end{array}$ \\
\hline & $\begin{array}{l}\text { Organo- } \\
\text { mineral } \\
\text { layers } \\
\text { horizons) }\end{array}$ & 4 & $\begin{array}{l}\text { Trace } \\
\mathrm{s}\end{array}$ & 0.3 & nd & 23 & \\
\hline \multirow{4}{*}{$\begin{array}{l}\text { Draix } \\
\text { catchment } \\
\text { (Haute- } \\
\text { Provence, } \\
\text { France) }\end{array}$} & $\begin{array}{l}\text { Recent soil } \\
\text { layers }\end{array}$ & & & & & & \\
\hline & $\begin{array}{l}\text { Fresh litters } \\
\text { (OL horizons) }\end{array}$ & 4 & 0 & 4.7 & 0.3 & 4 & $\begin{array}{l}\text { Di } \\
\text { Giovanni } \\
\text { (not } \\
\text { published } \\
\text { ) }\end{array}$ \\
\hline & $\begin{array}{l}\text { Humic layers } \\
\text { (OH } \\
\text { horizons) }\end{array}$ & 5 & 4 & 0.3 & 0.4 & 15 & \\
\hline & Organo- & 4 & 83 & 0.1 & 2.4 & 6 & \\
\hline
\end{tabular}




\begin{tabular}{|c|c|c|c|c|c|c|c|}
\hline \multirow[t]{2}{*}{ Location } & \multirow{2}{*}{$\begin{array}{l}\text { Sample } \\
\text { desc ription }\end{array}$} & \multirow[t]{2}{*}{$\mathbf{n}$} & \multicolumn{4}{|c|}{ Optical indices } & \multirow{2}{*}{$\begin{array}{l}\text { Referenc } \\
\text { es }\end{array}$} \\
\hline & & & $\begin{array}{l}\text { AOM } \\
\text { (\%) }\end{array}$ & $\begin{array}{l}\text { Presenved } \\
\text { / } \\
\text { transforme } \\
\text { d }\end{array}$ & $\begin{array}{l}\text { Gelified/amorpho } \\
\text { us }\end{array}$ & $\begin{array}{l}\text { OP } \\
\text { (\%) }\end{array}$ & \\
\hline & $\begin{array}{l}\text { mineral } \\
\text { layers } \\
\text { horizons) }\end{array}$ & & & & & & \\
\hline \multirow{8}{*}{$\begin{array}{l}\text { Annecy } \\
\text { catchment } \\
\text { (French Alps) }\end{array}$} & $\begin{array}{l}\text { Recent soil } \\
\text { layers }\end{array}$ & & & & & & \\
\hline & $\begin{array}{l}\text { Organic } \\
\text { layers (OL, } \\
\text { OF and } \mathrm{OH} \\
\text { horizons) }\end{array}$ & $\begin{array}{l}1 \\
3\end{array}$ & $\begin{array}{l}\text { trace } \\
\mathrm{s}\end{array}$ & 2.6 & 0.2 & 2 & \\
\hline & $\begin{array}{l}\text { Organo- } \\
\text { mineral } \\
\text { layers } \\
\text { horizons) }\end{array}$ & $\begin{array}{l}1 \\
3\end{array}$ & $\begin{array}{l}\text { trace } \\
\mathrm{s}\end{array}$ & 0.8 & 0.1 & 7 & \\
\hline & $\begin{array}{l}\text { Late } \\
\text { Holocene } \\
\text { lacustrine } \\
\text { deposits }\end{array}$ & & & & & & \\
\hline & $\begin{array}{l}\text { Micritic } \\
\text { limestone }\end{array}$ & $\begin{array}{l}1 \\
3\end{array}$ & 43 & 0.4 & 0.4 & 6 & $\frac{\text { Noël et }}{\text { al., 2001 }}$ \\
\hline & $\begin{array}{l}\text { Micritic } \\
\text { limestone } \\
\text { with some } \\
\text { detrital } \\
\text { events }\end{array}$ & $\begin{array}{l}7 \\
5\end{array}$ & 30 & 0.3 & 0.2 & 14 & \\
\hline & $\begin{array}{l}\text { Micritic } \\
\text { limestone } \\
\text { with many } \\
\text { detrital } \\
\text { events }\end{array}$ & $\begin{array}{l}6 \\
6\end{array}$ & 31 & 0.7 & 1.5 & 18 & \\
\hline & $\begin{array}{l}\text { Dark organic } \\
\text { mud (gyttja) }\end{array}$ & $\begin{array}{l}4 \\
9\end{array}$ & 52 & 1.3 & 1 & 9 & \\
\hline \multirow[t]{2}{*}{$\begin{array}{l}\text { Tritrivakely } \\
\text { marsh } \\
\text { (Madagasc } \\
\text { ar) }\end{array}$} & $\begin{array}{l}\text { Late } \\
\text { Holocene } \\
\text { peaty } \\
\text { deposits }\end{array}$ & & & & & & \\
\hline & $\begin{array}{l}\text { Palustrine } \\
\text { peaty } \\
\text { deposits }\end{array}$ & $\begin{array}{l}1 \\
0\end{array}$ & 15 & 0.9 & 0.3 & 5 & $\begin{array}{l}\frac{\text { Bourdon }}{\text { et al.. }} \\
\underline{2000}\end{array}$ \\
\hline
\end{tabular}




\begin{tabular}{|c|c|c|c|c|c|c|c|}
\hline \multirow[t]{2}{*}{ Location } & \multirow{2}{*}{$\begin{array}{l}\text { Sample } \\
\text { desc niption }\end{array}$} & \multirow[t]{2}{*}{$\mathbf{n}$} & \multicolumn{4}{|c|}{ Optical indices } & \multirow{2}{*}{$\begin{array}{l}\text { Referenc } \\
\text { es }\end{array}$} \\
\hline & & & $\begin{array}{l}\text { AOM } \\
\text { (\%) }\end{array}$ & $\begin{array}{l}\text { Preserved } \\
\text { / } \\
\text { transforme } \\
\text { d }\end{array}$ & $\begin{array}{l}\text { Gelified/amorpho } \\
\text { us }\end{array}$ & $\begin{array}{l}\text { OP } \\
\text { (\%) }\end{array}$ & \\
\hline & $\begin{array}{l}\text { Lacustrine } \\
\text { peaty } \\
\text { deposits }\end{array}$ & $\begin{array}{l}1 \\
0\end{array}$ & 30 & 0.7 & 0.1 & 10 & \\
\hline
\end{tabular}

$\mathrm{n}$ : number of samples.

\subsection{Application of optical indices to Holocene deposits}

The four new indices presented in this paper were used to characterize the Holocene fluvio-palustrine deposits cored in the Lower Seine Valley (Table 4). Sixty-three samples were analysed and were found to be distributed within fields defined in Fig. 6 according to the nature of OM (Fig. 7a) and the origin of the terrestrial fraction (Fig. 7b). Although environments do not exactly correspond to those of the Vernier Marsh, the different optical signatures allow one to discuss the sedimentary OM origin.

Table 4. : Lithology and optical indices of Holocene fluvio-palustrine deposits cored in the Lower Seine Valley (Fig. 1b)

\begin{tabular}{|l|l|l|l|l|l|l|}
\hline Samples & \multicolumn{7}{|l|}{ Optical compositional indices } \\
\hline Lithology & $\begin{array}{l}\text { Depth } \\
\text { (cm) }\end{array}$ & $\begin{array}{l}\text { TOC } \\
\text { (\%) }\end{array}$ & $\begin{array}{l}\text { AOM } \\
\text { (\%) }\end{array}$ & $\begin{array}{l}\text { Presenved/ } \\
\text { transfomed }\end{array}$ & $\begin{array}{l}\text { Gelified/ } \\
\text { amorphous }\end{array}$ & $\begin{array}{l}\text { OP } \\
\text { (\%) }\end{array}$ \\
\hline $\begin{array}{l}\text { Clayey } \\
\text { loam }\end{array}$ & 1 & - & 69 & 0.58 & 3.33 & 6 \\
\hline & 11 & 2.3 & 89 & 0.03 & 4.00 & 5 \\
\hline & 21 & 1.8 & 90 & 0.20 & 3.00 & 3 \\
\hline $\begin{array}{l}\text { Organic } \\
\text { mud }\end{array}$ & 31 & 2.9 & 80 & 0.12 & 0.50 & 2 \\
\hline & 51 & 17.6 & 4 & 0.08 & 0.83 & 2 \\
\hline & 59 & 10.9 & 23 & 0.12 & 0.86 & 2 \\
\hline Sandy loam & 67 & 0.5 & 15 & 0.40 & 1.32 & 2.22 \\
\hline
\end{tabular}




\begin{tabular}{|c|c|c|c|c|c|c|}
\hline \multicolumn{3}{|l|}{ Samples } & \multicolumn{4}{|c|}{ Optical compositional indices } \\
\hline Lthology & $\begin{array}{l}\text { Depth } \\
\text { (cm) }\end{array}$ & $\begin{array}{l}\text { TOC } \\
\text { (\%) }\end{array}$ & $\begin{array}{l}\text { AOM } \\
\text { (\%) }\end{array}$ & $\begin{array}{l}\text { Preserved/ } \\
\text { transformed }\end{array}$ & $\begin{array}{l}\text { Gelified/ } \\
\text { amorphous }\end{array}$ & $\begin{array}{l}\text { OP } \\
\text { (\%) }\end{array}$ \\
\hline & 95 & 0.7 & 1 & 0.19 & 2.60 & 48 \\
\hline & 105 & 0.5 & 1 & 0.28 & 1.07 & 16 \\
\hline \multirow{7}{*}{$\begin{array}{l}\text { Clayey } \\
\text { peat }\end{array}$} & 125 & 17.3 & 1 & 0.45 & 0.34 & 3 \\
\hline & 135 & 16.8 & 0 & 0.39 & 0.60 & 2 \\
\hline & 145 & 14.4 & 0 & 0.59 & 0.61 & 3 \\
\hline & 159 & 15.7 & 0 & 0.46 & 0.33 & 4 \\
\hline & 167 & 16.1 & 0 & 0.66 & 0.64 & 4 \\
\hline & 177 & 16.4 & 0 & 0.55 & 0.53 & 2 \\
\hline & 197 & 18.2 & 1 & 0.34 & 0.50 & 1 \\
\hline \multirow[t]{8}{*}{ Peat } & 207 & 36.9 & 0 & 0.12 & 1.21 & 2 \\
\hline & 227 & 33.3 & 0 & 0.57 & 0.41 & 2 \\
\hline & 247 & 39.5 & 1 & 0.94 & 0.38 & 3 \\
\hline & 267 & 40.1 & 0 & 0.52 & 0.85 & 1 \\
\hline & 277 & 37.7 & 0 & 0.62 & 0.55 & 2 \\
\hline & 287 & 39.0 & 0 & 1.18 & 0.69 & 1 \\
\hline & 307 & 33.1 & 0 & 0.76 & 0.86 & 1 \\
\hline & 317 & 31.8 & 1 & 0.40 & 0.56 & 1 \\
\hline \multirow{2}{*}{$\begin{array}{l}\text { Organic } \\
\text { mud }\end{array}$} & 327 & 10.3 & 1 & 0.49 & 0.75 & 10 \\
\hline & 337 & 10.8 & 0 & 1.16 & 1.29 & 4 \\
\hline \multirow[t]{5}{*}{ Peat } & 347 & 27.8 & 0 & 0.53 & 0.63 & 5 \\
\hline & 367 & 34.8 & 0 & 0.40 & 0.88 & 4 \\
\hline & 377 & 39.1 & 0 & 0.67 & 0.42 & 3 \\
\hline & 397 & 27.4 & 0 & 0.42 & 0.78 & 2 \\
\hline & 407 & 41.4 & 0 & 0.47 & 0.63 & 1 \\
\hline
\end{tabular}




\begin{tabular}{|l|l|l|l|l|l|l|}
\hline Samples & \multicolumn{7}{l|}{ Optical compositional indices } \\
\hline Lithology & $\begin{array}{l}\text { Depth } \\
\text { (cm) }\end{array}$ & $\begin{array}{l}\text { TOC } \\
\text { (\%) }\end{array}$ & $\begin{array}{l}\text { AOM } \\
\text { (\%) }\end{array}$ & $\begin{array}{l}\text { Presenved/ } \\
\text { transformed }\end{array}$ & $\begin{array}{l}\text { Gelified/ } \\
\text { amonhous }\end{array}$ & $\begin{array}{l}\text { OP } \\
\text { (\%) }\end{array}$ \\
\hline & 417 & 34.5 & 1 & 0.38 & 0.43 & 2 \\
\hline Silty sands & 425 & 5.6 & 1 & 0.63 & 0.35 & 2 \\
\hline & 435 & 1.3 & 1 & 0.50 & 0.26 & 13 \\
\hline & 443 & - & 16 & 0.68 & 0.57 & 6 \\
\hline & 455 & 2.6 & 18 & 0.66 & 0.67 & 1.06 \\
\hline & 477 & - & 18 & 0.48 & 0.37 & 13 \\
\hline & 485 & 3.2 & 6 & 0.80 & & 7 \\
\hline
\end{tabular}

Basal grey silty sands present a signature comparable to that of modern soils (i.e. litter and histosols; Fig. 7) relative to the "preserved / transformed ratio" $(>0.5$ and <0.8) and "GP / AP ratio" $>0.3$ and < 1.1). These older fluvial deposits are interpreted to be related to braided channels where sediments included materials derived from nearly soils that were developed in a forested context based on sedimentological and paleobiological observations (Sebag, 2002). In this case, the amorphous particles represent a paraautochthonous fraction (i.e. reworked from surrounding forested litters). On the other hand, some layers in the basal silty sand seem relatively enriched in aquatic OM ("AOM contents" > 15\%) and in strongly oxidized allochthonous particles ("OP contents" > 10\%). The main sedimentary body is composed of several meters of peat and clayey peat related to an alluvial backswamp system that was sometimes open to sediment contributions from the Seine River (Sebag, 2002). These deposits present an optical signature that was comparable to that of modern histosols (Fig. 7). The main variations depend on the state and condition of degradation $(0.3<$ "preserved / transformed ratio" < 1.2; $0.4<$ "GP / AP ratio" < 1.5) of the terrestrial fraction. They could be related to local hydrological fluctuations, which controlled the diagenesis of the autochthonous terrestrial fraction. When the groundwater level is low, phytoclast biodegradation is favoured (low values of "preserved / transformed ratio") as is the formation of amorphous particles (low values of "GP / AP ratio") involving aerobic processes. When the groundwater level is high, biodegradation is slowed (high values of "preserved / transformed ratio") whereas more anaerobic processes favoured gelification of plant tissues (high values of "GP / AP ratio"). 


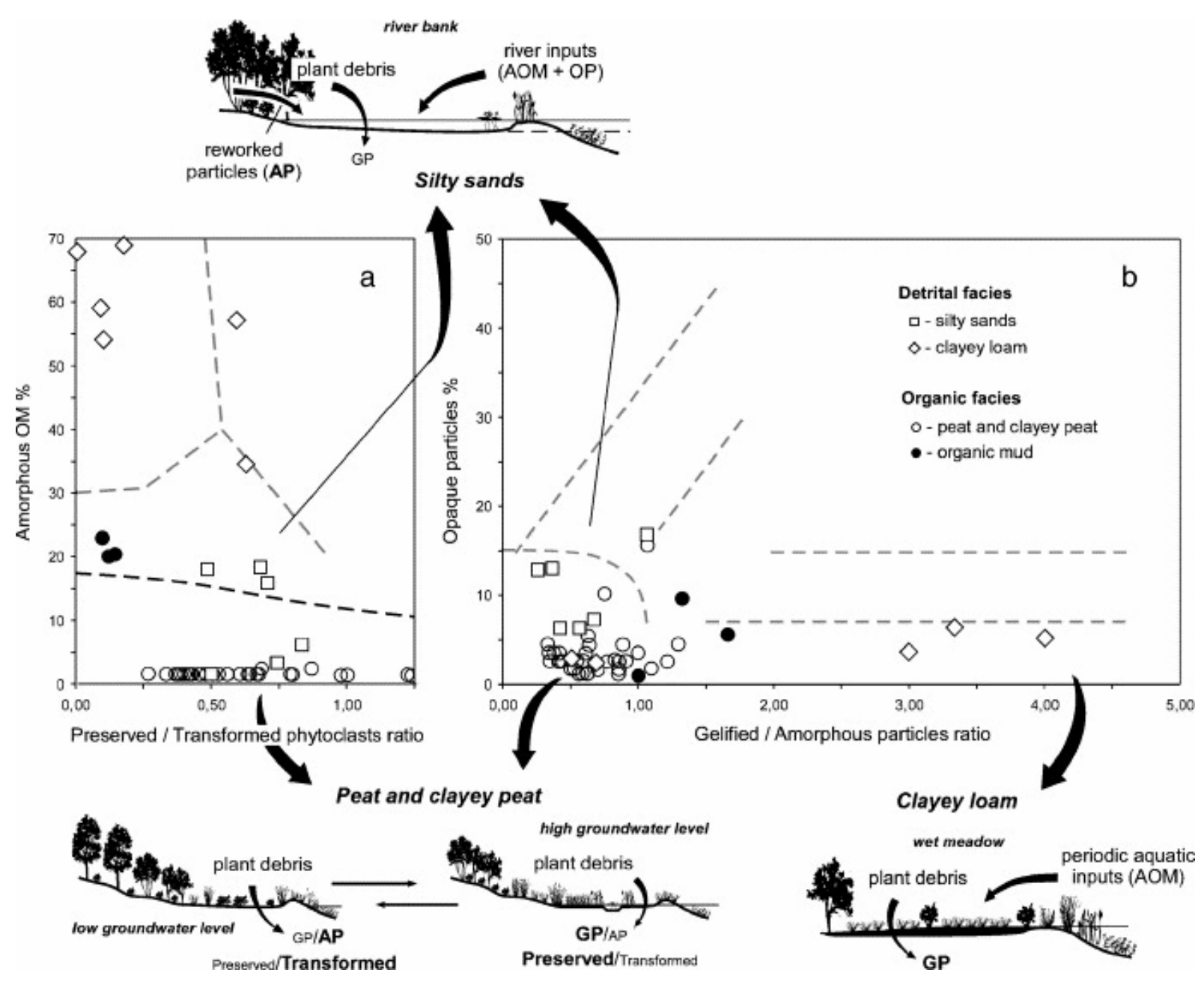

Fig. 7. Characterization of Holocene deposits cored in the Lower Seine Valley with four optical indices (see text). (a) Nature of OM by using "AOM contents" (aquatic or microbial production) and "preserved / transformed phytoclasts ratio" (degree of preservation of terrestrial plant debris). (b) Origin of terrestrial fraction by using "OP contents" (allochthonous fraction from fluvial origin) and "GP / AP ratio" (pedogenic or subaquatic degradation of terrestrial plant debris).

Surficial deposits from the Lower Seine Valley correspond to recent alluvial clayey loams on which wet meadows develop (Sebag, 2002). These layers have high "AOM contents" (>30\%), with some OM of aquatic origin (alluvial deposits). However, the relatively advanced state of degradation and the lack of oxidized particles (i.e. opaque particles) give evidence of the origin of the terrestrial fraction. These characteristics are not linked to an allochthonous fraction transported by water, but are associated with post-sedimentary contributions related to a plant cover developed after deposition.

In this one example, the first two indices ("AOM contents" and "preserved / transformed ratio"; Fig. 7a) allow one to distinguish three distinct organic assemblages (i.e. terrestrial, mixed or aquatic $O M$ ) which are in agreement with the sedimentary record. The "OP contents" show a palustrine signature $(\mathrm{OP}<10 \%)$ or a mixed signature (OP $>10 \%)$. The palustrine samples 
are distributed in two fields: (1) a homogeneous group (strictly terrestrial supplies: GP / AP < 1.5) and (2) another group corresponding to various GP / AP values (wet meadows: GP / AP > 1.5). Within each group, the variations of both ratios could be interpreted in terms of depositional environment (i.e. fluctuations of local hydrological conditions).

\section{Conclusion}

The various optical markers are classically used to study depositional systems that are marked by distinct organic sources. Indeed in the lakes or peaty environments, both autochthonous and allochthonous materials are easily characterized by the results of the palynofacies' analysis. On the other hand, in riparian wetlands, both autochthonous and allochthonous OM sources may be derived from marsh vegetation, fringe forests, algal vegetation, riverine transport of eroded soils and aquatic organisms. Using optical compositional indices allows one to characterize sedimentary organic contents and to compare obviously different samples. In the present study, the results are interpreted in terms of $O M$ origin (i.e. aquatic or terrestrial), source (i.e. autochthonous, para-autochthonous or allochthonous) and diagenesis (i.e. aerobic or anaerobic). This approach could be used to study both sub-recent (i.e. Holocene) and modern fluvio-palustrine deposits. Applied to existing wetlands, such as the Vernier Marsh, this approach leads us to an interpretation of the deposition and redeposition of $O M$ on a landscape scale. Such information is essential to correctly interpret bulk geochemical data that tend to obscure details by averaging results derived from mixed samples. In addition, this kind of study allows one to define a modern frame of reference that can be applied in paleoenvironmental reconstructions, as shown by the compositional indices used in the study of Holocene alluvial deposits.

\section{Acknowledgements}

This work is a contribution to the research programs of the UMR 6113 CNRS/ISTO and the Projet Collectif de Recherche "Les paysages et les hommes en basse vallée de la Seine depuis 10.000 ans" (UMR 6143 CNRS/M2C) funded by DRAC-Haute-Normandie and DIREN-HauteNormandie, by the Conseil Regional de Haute-Normandie, and by five mining companies (CBN, FCH, Larfarge Granulats, Morillon Corvol and STREF). We thank J.P. Bakyono for their assistance in laboratory treatments. We thank also E. Lallier-Vergès, S. Neuzil and F. Rich for their help, remarks and advice. 


\section{References}

Alpern, 1976 B. Alpern, Fluorescence et réflectance de la matière organique dispersée et évolution des sédiments, Bulletin du Centre de Recherches de Pau - SNPA 10 (1976), pp. 201-220.

Barber et al., 2003 K.E. Barber, F.M. Chambers and D. Maddy, Holocene palaeoclimates from peat stratigraphy: macrofossil proxy climate records from three oceanic raised bogs in England and Ireland, Quatemary Science Reviews 22 (2003), pp. 521-539.

Batten, 1996 D.J. Batten, Palynofacies and palaeoenvironmental interpretation. In: D.C. McGregor, Editor, Palynology: Principles and Applications, American Association of Stratigraphic Palynologists Foundation (1996), pp. 1011-1064.

Bourdon et al., 2000 S. Bourdon, F. Laggoun-Défarge, J.R. Disnar, O. Maman, B. Guillet, S. Derenne and C. Largeau, Organic matter sources and early diagenetic degradation in a tropical peaty marsh (Tritrivakely, Madagascar). Implications for environmental reconstruction during the Sub-Atlantic, Orga nic Geochemistry 21 (2000), pp. 421-438.

Buillit et al., 1997 N. Buillit, E. Lallier-Vergès, J.R. Disnar and J.L. Loizeau, Changements climatiques et effets anthropiques au cours du dernier millénaire attestés par l'étude pétrographique de la matière organique (Annecy, Le Petit Lac, France), Bulletin de la Société Géologique de France 168 (1997), pp. 573-583.

Clymo, 1983 R.S. Clymo, Peat. In: A.J.P. Goore, Editor, Mires: Swamp, Bog, Fen and Moor. Ec osystems of the World, Elsevier, Amsterdam (1983), pp. 159-224.

Cohen et al., 1999 A.D. Cohen, C.P. Gage and W.S. Moore, Combining organic petrography and palynology to assess anthropogenic impacts on peatlands: Part 1. An example from the northern Everglades of Florida, Intemational J oumal of Coal Geology 39 (1999), pp. 3-45.

Combaz, 1964 A. Combaz, Les palynofaciès, Revue de Micropaléontologie 7 (1964), pp. 205-218.

Di Giovanni et al., 1999a C. Di Giovanni, M. Campy and J.R. Disnar, Les particules charbonneuses, témoins des variations de l'érosion chimique d'un bassin versant calcaire durant l'Holocène (bassin de Chaillexon, Doubs, France), Bulletin de la Société géologique de France. Comptes rendus de l'Académie des sciences Paris 328 (1999), pp. 167-172.

Di Giovanni et al., 1999b C. Di Giovanni, M. Meybeck and H. Richard, Variations in sediment yield from the Upper Doubs River carbonate watershed 
(Jura, France) since the Late-Glacial Period, Quatemary Research 51 (1999), pp. 267-279.

Di Giovanni et al., 1999C C. Di Giovanni, J.R. Disnar, M. Campy and J.J. Macaire, Variability of the ancient organic supply in modern humus, Analusis 27 (1999), pp. 398-402.

Di Giovanni et al., 2000 C. Di Giovanni, J.R. Disnar, V. Bichet and M. Campy, Saisonnalité et effets de seuils de la sédimentation organique détritique en milieu lacustre: hétérochronie de l'enregistrement organique et des fluctuations climatiques (bassin de Chaillexon, Doubs, France), Bulletin de la Société Géologique de France 171 (2000), pp. 533-544.

Di Giovanni et al., 2002 C. Di Giovanni, J.R. Disnar and J.J. Macaire, Estimation of the annual yield of organic carbon released from carbonates and shales by chemical weathering, Global and Planetary Change 32 (2002), pp. 195210.

Gorham, 1991 E. Gorham, Northern peatlands: role in the carbon cycle and probable responses to climatic warming, Ecological Economics 1 (1991), pp. 182-195.

Laggoun-Défarge et al., 1995 F. Laggoun-Défarge, B. Pradier, E. Brosse, S. Belin and J.L. Oudin, Analyse microtexturale des sédiments organiques du Delta de la Mahakam (Indonésie), Relations avec les Environnements de Dépôt. Comptes rendus de l'Aca démie des sciences Paris 320 (1995), pp. 1055-1061.

Laggoun-Défarge et al., 1999 F. Laggoun-Défarge, S. Bourdon, B. Guillet and O. Maman, Nature and degradation mode of organic matter in Tritrivakely Peaty Marsh (Madagascar). Application to environmental reconstruction during the last 2300 years, Ecologie 30 (1999), pp. 65-68.

Lallier-Vergès et al., 1998 E. Lallier-Vergès, B.P. Perrussel, J.R. Disnar and F. Baltzer, Relationships between environmental conditions and diagenetic evolution of organic matter derived from higher plants in a modern mangrove swamp system (Guadeloupe, French West Indies), Organic Geochemistry 29 (1998), pp. 1663-1686.

Malmer, 1992 N. Malmer, Peat accumulation and the global carbon cycle. In: E. Koster, Editor, Greenhouse-Impact on Cold-Climate ecosystems and Landsca pes. Catena Supplement, LICC, Cremlingen (1992), pp. 97-1 10.

Mesnage et al., 2002 V. Mesnage, S. Bonneville, B. Laignel, D. Lefebvre, J.P. Dupont and D. Mikes, Filling of a wetland (Seine estuary, France): natural eutrophication or anthropogenic process? A sedimentological study of wetland organic sediments, Hydrobiologia 475/476 (2002), pp. 423-435. 
Meyers, 1997 P.A. Meyers, Organic geochemical proxies of paleoceanographic, paleolimnologic, and paleoclimatic processes, Organic Geochemistry 27 (1997), pp. 231-250.

Noël et al., 2001 H. Noël, E. Garbolino, A. Braver, E. Lallier-Vergès, J.L. de Beaulieu and J.R. Disnar, Human impact and soil erosion during the last 5000 yrs as recorded in lacustrine sedimentary organic matter at Lac d'Annecy, the French Alps, J oumal of Paleolimnology 25 (2001), pp. 229-244.

Patience et al., 1996 A.J. Patience, E. Lallier-Vergès, P. Alberic, A. Desprairies and N. Tribovillard, Relationships between organo-mineral supply and early diagenesis in the lacustrine environment: a study of surficial sediments from the Lac du Bouchet (Haute Loire, France), Quatemary Science Reviews 15 (1996), pp. 213-221.

Sebag, 2002 Sebag, D., 2002. Apports de la matière organique pour la reconstitution des paléoenvironnements holocènes de la basse vallée de la Seine. Fluctuations des conditions hydrologiques locales et environnements de dépôt. PhD thesis, Université de Roven, France.

Sifeddine et al., 1996 A. Sifeddine, P. Bertrand, E. Lallier-Vergès and A.J. Patience, Lacustrine organic fluxes and paleoclimatic variations during the last 15 ka: Lac du Bouchet (Massif Central, France), Quatemary Science Reviews 15 (1996), pp. 203-211.

Sifeddine et al., 1998 A. Sifeddine, J. Bertaux, P. Mourguiart, L. Martin, J.R. Disnar, F. Laggoun-Défarge and J. Argollo, Ełude de la sédimentation lacustre d'un site de forêt d'altitude des Andes centrales (Bolivie). Implications paléoclimatiques, Bulletin de la Société Géologique de France 169 (1998), pp. 395-402.

Tyson, 1995 R.V. Tyson, Sedimentary Organic Matter: Organic Facies and Palynofacies, Chapman and Hall, London (1995).

Whigham, 1999 D.F. Whigham, Ecological issues related to wetland preservation, restoration, creation and assessment, The Science of the Total Environment 240 (1999), pp. 31-40. 